\title{
Bidirectional Energy-based Pushover Procedure as a Fast Approach to Establish Approximate IDA Curves under Biaxial Seismic Excitations: An Evaluation for Medium- and High-rise Buildings
}

Sahman Soleimani ( $\nabla$ sahman.soleimani@griffthuni.edu.au )

Griffith University - Gold Coast Campus https://orcid.org/0000-0002-3827-5400

Abdolreza Sarvghad Moghadam

IIEES: International Institute of Earthquake Engineering and Seismology

Armin Aziminejad

Islamic Azad University Science and Research Branch

\section{Research Article}

Keywords: energy-based pushover analysis, biaxial seismic excitation, incremental dynamic analysis, asymmetric-plan buildings, medium and high-rise buildings

Posted Date: May 28th, 2021

DOl: https://doi.org/10.21203/rs.3.rs-467175/v1

License: (c) (1) This work is licensed under a Creative Commons Attribution 4.0 International License. Read Full License

Version of Record: A version of this preprint was published at Bulletin of Earthquake Engineering on January 31st, 2022. See the published version at https://doi.org/10.1007/s10518-022-01324-z. 


\title{
Bidirectional energy-based pushover procedure as a fast approach to establish approximate IDA curves under biaxial seismic excitations: an evaluation for medium- and high-rise buildings
}

Sahman Soleimani (corresponding author), School of Engineering and Built Environment, Griffith University, Queensland, Australia

Sahman.Soleimani@griffithuni.edu.au

Abdolreza S. Moghadam, International Institute of Earthquake Engineering and Seismology, Tehran, Iran

Moghadam@iiees.ac.ir

Armin Aziminejad, Science and Research Branch, Islamic Azad University, Tehran, Iran

ArminAziminejad@srbiau.ac.ir

\begin{abstract}
Bidirectional energy-based pushover (BEP) procedure is expanded in this paper to predict approximate incremental dynamic analysis (IDA) results of medium- and high-rise structures. BEP is a unique approach in the sense that it provides approximate IDA curves under the simultaneous effect of two horizontal components of ground motions and is applicable to both symmetric- and asymmetric-plan buildings. The method has already proved to be useful in low-rise buildings, and this study aims to evaluate its suitability for mid- and high-rise structures. Six structural models were considered in this evaluation in two groups of 9- and 20-story buildings, with each group consisting of a symmetric, a one-way asymmetric, and a two-way asymmetric-plan building. The results revealed that the method was sufficiently accurate to provide approximate IDA curves for all structural models. The method had similar accuracy in the asymmetric models as it did in the symmetric models, although the accuracy slightly decreased as the height of the building increased. BEP also provided good estimates of the demands in both 'flexible' and 'stiff sides' of the asymmetric buildings as well as the demands over the height of the buildings.
\end{abstract}

Keywords: energy-based pushover analysis, biaxial seismic excitation, incremental dynamic analysis, asymmetricplan buildings, medium and high-rise buildings

\section{Declarations}

Funding: No external support was received in this research. The funding was provided by the authors.

\section{Conflicts of interest/Competing interests: N/A}

Availability of data and material: the data used in this research is transparent and valid references have been made when applicable. 
Code availability: Custom codes are used in this research, which have not been made public.

\section{Ethics approval: N/A}

Consent to participate: Informed consent was obtained from all individual participants included in the study.

Consent for publication: All information in this paper, including the text and figures, has been produced by the authors, and I give my consent for the publication of this manuscript in "Bulletin of Earthquake Engineering."

\section{Introduction}

Incremental dynamic analysis (IDA) is a parametric analysis method used for seismic assessment of structures (Vamvatsikos and Cornell 2002). IDA involves a structural model being subjected to an ensemble of ground motion records, each scaled to multiple intensity levels, covering a wide range of structural behavior from elastic domain to global instability. The results of IDA are generally presented as curves of maximum responses parameterized versus the intensity measure.

Since IDA entails numerous nonlinear dynamic analyses (NDA), the computational burden involved is known to be the main disadvantage to this method, especially in tall buildings comprising a large number of nonlinear structural elements. Hence, approximate procedures have been proposed in the literature aiming at reducing the computational cost associated with IDA.

By exploring the relationship between the static pushover (SPO) and the IDA outcomes through parametric studies, Vamvatsikos and Cornell (2005) developed the SPO2IDA method to predict approximate 16th, 50th, and 84th percentile IDA curves. The procedure was recently employed to produce structure-specific fragility curves (Baltzopoulos, Baraschino et al. 2017). Designed for the first-mode dominated structures, SPO2IDA is not expected to maintain high levels of accuracy in high-rise or asymmetric buildings. Han and Chopra (2006) proposed an incremental version of the well-known modal pushover analysis (MPA) (Chopra and Goel 2002) to produce approximate IDA curves. This method has the advantage to consider the contribution of higher modes. However, the distortions occasionally encountered in the higher-mode capacity curves in this procedure often cause disruption in the process of MPA (FEMA 2005). This is especially the case when the building is subjected to ground motion records causing significant nonlinearity in higher modes. Brozovič and Dolšek (2014) presented the envelope-based pushover analysis in which a pushover analysis is conducted for each mode, and the total demands are determined by enveloping the results obtained from all pushover analyses performed. Other methods have also been presented in 
the literature, each taking a different strategy to alleviate the computational demand of IDA (Azarbakht and Dolšek 2007, Zarfam and Mofid 2011, Peruš, Klinc et al. 2013)

Among these methods, a few have addressed asymmetric-plan buildings. Dolšek and Fajfar (2007) evaluated the incremental N2 method on a three-story asymmetric reinforced concrete building and concluded that the method is viable in assessing plan-asymmetric buildings. Based on the 2DMPA method (Lin and Tsai 2007), Birzhandi and Halabian (2017) developed an approximate IDA procedure for plan-asymmetric structures, allowing for the hysteresis characteristics of structures such as pinching, strength loss, and stiffness deterioration. The authors later extended the method to take soil-structure interactions into account $(2018,2020)$.

The only approximate IDA method to address biaxial seismic excitations in asymmetric-plan buildings was "bidirectional energy-based pushover" (BEP) (Soleimani, Aziminejad et al. 2018). While symmetric buildings can be assessed independently along $\mathrm{x}$ - and $\mathrm{y}$-directions, it is essential for asymmetric-plan buildings to be evaluated under both components of ground motions, simultaneously. This is because the two horizontal components could neutralize or amplify each other's effect when applied simultaneously. The BEP formulation addresses this issue as it allows for both components of ground motions to be considered at the same time.

The BEP method can be summarized in three steps. Firstly, modal capacity curves-i.e., the force-deformation relationships for modal single-degree-of-freedom (SDF) systems - are established from energy-based modal pushover analyses. Secondly, each SDF system is subjected to a combination of the two horizontal components of the ground motion, and the peak responses are extracted. Finally, the corresponding structural responses are combined using a modified CQC formula.

The energy-based pushover approach employed in the BEP is a technique to prevent distortions of capacity curves. The conventional pushover methods use the roof displacement during pushover analysis as an index to derive the displacement of the corresponding SDF system. The same goal is achieved in the energy-based pushover using the absorbed energy rather than roof displacement throughout the pushover analysis. The method was first proposed by Hernandez-Montes, Kwon et al. (2004) for two-dimensional structures and extended to asymmetric-plan buildings by Soleimani, Aziminejad et al. (2017). The energy-based pushover also found application in the assessment of structures exposed to other load scenarios rather than earthquakes (Saedi Daryan, Soleimani et al. 2017, 2018).

The usefulness of BEP has been demonstrated in the previous research (Soleimani, Aziminejad et al. 2018) through the assessment of a three-story asymmetric building - around four percent error in prediction of the median IDA curves. However, its suitability for taller buildings is still unknown. While the first triple modes are adequate to estimate the demands of low-rise buildings, the dynamic response of tall buildings is strongly influenced by higher modes, which pose a challenge to BEP. When the plan is asymmetric, the torsional motions are also involved, adding to the complexity of the structural behavior.

This study aims to evaluate BEP for medium- and high-rise buildings. The influence of asymmetry on the accuracy of the results is also assessed. First, a detailed step-by-step procedure to perform BEP is presented. Then, the 
structural models and ground motions used in this evaluation are introduced. The structural models are defined in two groups of 9- and 20-story buildings, with each group comprising a symmetric, a one-way asymmetric, and a two-way asymmetric model. The models are chosen in this way so as to provide an insight into the effects of the building height and asymmetry on the accuracy of predicted IDA curves. Finally, the results of BEP are presented in comparison with those of the exact IDA. The accuracy of the method in predicting the maximum demands over the plan of the asymmetric models is evaluated (i.e., 'flexible side', mass center (CM), and 'stiff side'). Also, the driftratio demands over the height of the buildings are covered.

\section{Step by step methodology}

The BEP procedure is carried out through the step-by-step methodology presented in this section. More information can be found in Soleimani, Aziminejad et al. (2018).

1- Create a nonlinear 3D model of the building.

2- Calculate the natural frequencies, $\omega_{n}$, and the modes of natural vibration, $\boldsymbol{\varphi}_{n}$, at the elastic state of the building by running an eigenvalue analysis.

3- Apply gravity loads; then conduct the nth-mode pushover analysis using $\boldsymbol{m} \boldsymbol{\varphi}_{n}$ as the lateral load pattern. The term $\boldsymbol{m}$ is the matrix of mass.

4- Extract the nth-mode capacity curve (i.e., $\alpha_{n}-y_{n}$ curve) from the corresponding pushover analysis carried out in step 3. To do so, first, determine $\alpha_{n}$ and $y_{n}$, representing the resisting force and the displacement of the nthmode SDF system, respectively, throughout the pushover analysis; then combine the two parameters into a $\alpha_{n}-y_{n}$ curve. To determine $\alpha_{n}$ and $y_{n}$, follow the subsequent sub-steps:

4-1) Depending on the available component of the base shear, choose one of the following formulas to calculate $\alpha_{n}:$

$$
\alpha_{n}=\frac{V_{n x}}{L_{n x}} \quad \alpha_{n}=\frac{V_{n y}}{L_{n y}}
$$

in which $V_{n x}$ and $V_{n y}$ are the x- and y-components of the base shear throughout the nth-mode pushover analysis, and

$$
\begin{array}{ll}
L_{n x}=\boldsymbol{\varphi}_{n}^{T} \boldsymbol{m} \boldsymbol{l}_{x} & L_{n y}=\boldsymbol{\varphi}_{n}^{T} \boldsymbol{m} \boldsymbol{l}_{y} \\
\boldsymbol{l}_{x}=(\mathbf{1 , 0 , 0})^{\mathrm{T}} & \boldsymbol{l}_{y}=(\mathbf{0 , 1 , 0})^{\mathrm{T}}
\end{array}
$$

The terms $\mathbf{1}$ and $\mathbf{0}$ are $1 \times N$ vectors with all elements equal to unity and zero, respectively, and $N$ is the number of floors. It should be mentioned that if the building is two-way asymmetric, any of the two formulas in Eq. ( 2 ) can be used to calculate $\alpha_{n}$ since all modes would normally include both $\mathrm{x}$ - and y-components. Otherwise, the formula needs to be chosen so that the associated component of the base shear is nonzero. 
Calculate $y_{n}$ using the following formula:

$$
y_{n}=\int_{0}^{T} d y_{n}=\int_{0}^{T} \frac{d E_{n}}{\alpha_{n} M_{n}}
$$

in which $T$ is the final step of pushover analysis, and $M_{n}$ represents the modal mass of the nth-mode ( $M_{n}=\boldsymbol{\varphi}_{n}^{T} \boldsymbol{m} \boldsymbol{\varphi}_{n}$ ). The term $d E_{n}$ is the energy absorbed by structure at each step of pushover analysis. It is calculated by multiplying the pushover load, which involves the lateral forces and torques, by the structure displacement at that step:

$$
d E_{n}=\left(\boldsymbol{m} \varphi_{n}\right)^{\mathrm{T}} d \boldsymbol{u}_{n}
$$

where $\boldsymbol{u}$ is the displacement vector with the order of $1 \times 3 N$.

5- Establish the nth-mode hysteresis curve by, first, idealizing the $\alpha_{n}-y_{n}$ curve as a multi-linear curve, then defining the unloading and reloading branches given the martial properties and the structural system. In this study, capacity curves are idealized by bilinear curves.

6- Repeat steps 3-5 for as many modes as needed to achieve a sufficient degree of accuracy, and save the results for the subsequent steps.

7- Choose a pair of orthogonal ground-acceleration records.

8- Choose a scale factor for the first stage of the BEP analysis, and calculate the 'effective dynamic force' of the nth-mode SDF system for all the included modes using the following formula:

$$
q_{n}(t)=-S F\left(\Gamma_{n x} \frac{K_{g x}}{g y}\right)
$$

In Eq. ( 5 ), $S F$ stands for the scale factor. The terms and represent x-and y-components of the ground acceleration record, and

$$
\Gamma_{n x}=\frac{L_{n x}}{M_{n}} \quad \Gamma_{n y}=\frac{L_{n y}}{M_{n}}
$$

9- Having the hysteresis function and the 'effective dynamic force', solve the differential equation of motion for the nth-mode SDF system [Eq. ( 7 )] using one of the existing time-stepping methods [chapter 5-(Chopra 2011)], and determine the peak response, $y_{n o}$.

$$
(t)+2 \zeta_{n} \omega_{n} \&(t)+\alpha_{n}(t)=q_{n}(t)
$$

The term $\zeta_{n}$ is the damping ratio of the nth-mode. 
10- Extract the structural responses associated with $y_{n o}$ from the nth-mode pushover analysis database established in step 3 .

11- For all the included modes, repeat the steps 9-10, and combine the results using the modified CQC formula:

$$
r_{o}=\left(\sum_{i=1}^{X} \sum_{j=1}^{X} \eta_{i j} \rho_{i j} r_{i o} r_{j o}\right)
$$

In this equation, $X$ stands for the number of modes involved. The terms $r_{i o}$ and $r_{j o}$ are the peak structural responses associated with $i$ th and $j$ th modes. The term $\rho_{i j}$ is the correlation coefficient between the $i$ th and $j$ th modes in terms of modal characteristics, and it is obtained from the following equation [Chapter 13-(Chopra 2011)]:

$$
\rho_{i j}=\frac{8 \sqrt{\zeta_{i} \zeta_{j}}\left(\beta_{i j} \zeta_{i}+\zeta_{j}\right) \beta_{i j}^{3 / 2}}{\left(1+\beta_{i j}^{2}\right)^{2}+4 \xi_{i} \xi_{j} \beta_{i j}\left(1+\beta_{i j}\right)^{2}+4\left(\zeta_{i}^{2}+\zeta_{j}^{2}\right) \beta_{i j}^{2}}
$$

where $\beta_{i j}=\omega_{i} / \omega_{j}$. The term $\eta_{i j}$, which has been added to the standard CQC formula for the BEP method, represents the correlation between two modes in terms of their 'effective dynamic forces,' and it is computed by the following formula:

$$
\eta_{i j}=\frac{\operatorname{cov}\left(q_{i}(t), q_{j}(t)\right)}{\sqrt{\operatorname{var}\left(q_{i}(t)\right) \operatorname{var}\left(q_{j}(t)\right.}}=\frac{\int_{0}^{\tau} q_{i}(t) q_{j}(t) d t}{\sqrt{\int_{0}^{\tau}\left(q_{i}(t)\right)^{2} \int_{0}^{\tau}\left(q_{j}(t)\right)^{2} d t}}
$$

where $\tau$ is the final stage of the ground acceleration time-history. It should be noted that the CQC rule is not used in its original form in BEP, because CQC is originally designed to combine modal structural responses when all modes are subjected to the same ground acceleration record. In BEP, however, each mode is subjected to a combination of the $\mathrm{x}$ - and $\mathrm{y}$-components of ground accelerations, which vary from mode to mode.

Therefore, the coefficient $\eta_{i j}$ is added to the standard CQC formula to take into account the correlations between the 'effective dynamic forces' of different modes.

12- Choose another $S F$ and redo steps 8-11 until $r_{o}$ exceeds the maximum capacity of the corresponding component in the structure.

13- Pick another pair of ground acceleration records and redo steps 7-12. 


\section{Mathematical models}

\subsection{Structural systems}

Six structural models were considered for this study in two sets of 9- and 20-story buildings. The first model in each set is the symmetric one, obtained from the SAC Steel Project for Los Angeles, California (Gupta and Krawinkler 1999). The second model is a variation of the first model with $15 \%$ eccentricity along the y-axis: the CM was moved from the geometric center as much as $15 \%$ of the plan dimension in the y-direction. The third model is another variation of the first model with $15 \%$ eccentricity along both $\mathrm{x}$ - and $\mathrm{y}$-axes: $\mathrm{CM}$ was moved as much as $15 \%$ of the plan dimension in each direction. These models are referred to in this paper with the letter $\mathrm{M}$ followed by the height of the building, followed by the model number: M91, M92, M93 for the 9-story buildings, and M201, M202, M203 for the 20-story buildings.

Fig. 1 shows the plans of the 9- and 20-story buildings. The CMs of the three models in each set are represented in the plans. The solid and dashed lines indicate the moment-resisting frames (MRFs) and the gravity frames, respectively, with the columns being located at the intersection of the lines. The 9- and 20-story buildings are designed with a single-level and two-level basement, respectively. In the 9-story buildings, the columns at the corner of the building have moment-resisting connections to the beams of only one side so as to avoid biaxial bending. In the 20-story buildings, however, all the exterior connections are designed as moment resistant, with box columns at the corners to resist biaxial bending. The design details (loading information, member sections, yield strength of members, etc.) can be found in Gupta and Krawinkler (1999).

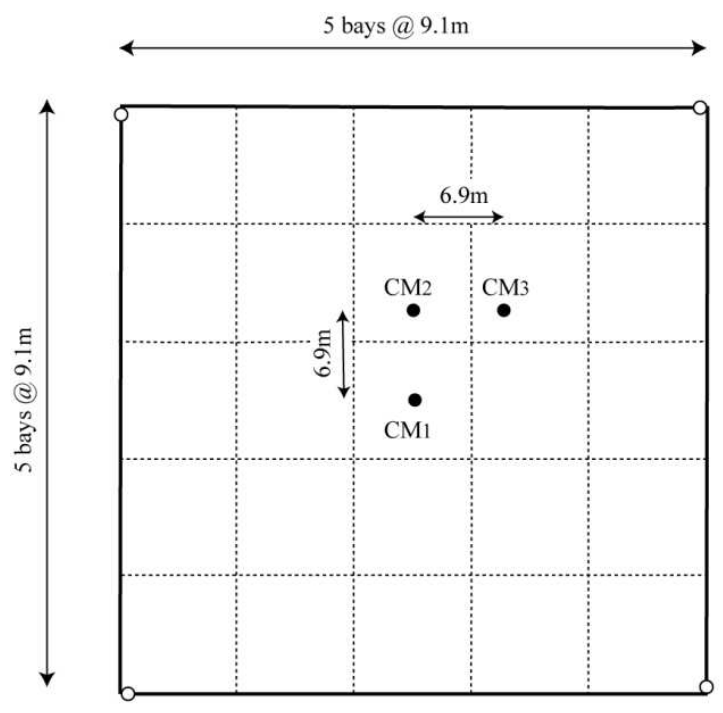

a)

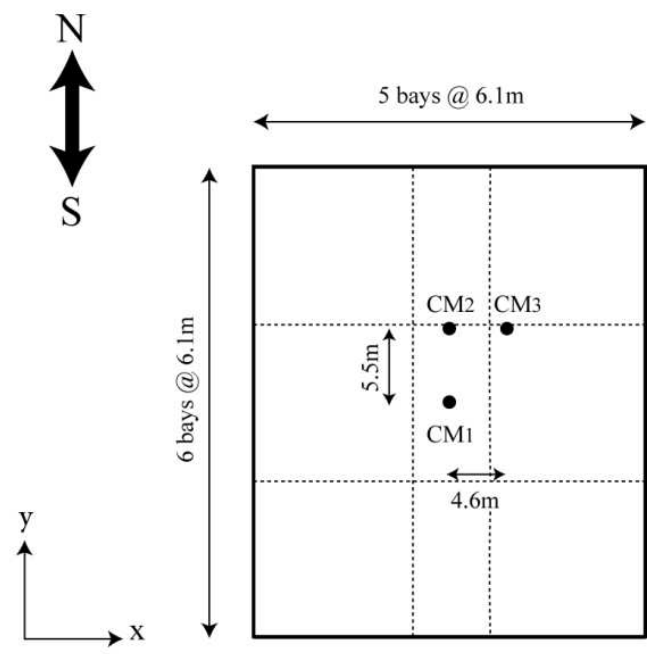

b)

Fig. 1. The plans of a) 9-story and b) 20 -story structural models. The points indicated by CM1, CM2 and CM3 are the mass centers of different models in each category. Solid lines represent moment resisting frames. 


\subsection{Modeling}

The mathematical models were created in PERFORM 3D (CSI 2011), by which the NDA and pushover analyses were carried out. The BEP analyses were performed using computer codes written in MATLAB in conjunction with PERFORM 3D. The mathematical models consisted of gravity columns modeled as linear along with MRFs modeled as nonlinear. The nonlinear properties of beam and columns (i.e., moment-rotation and the axial forcedeformation relationships) were defined in accordance with ASCE 41-13. The maximum plastic hinge rotation was presumed to be equal to $11 \theta_{y}$ as per ASCE 41-13, with $\theta_{y}$ being the yield rotation; any component exceeding this threshold was equated with the collapse of the building. Nonlinear hinges were defined as elastic perfectly-plastic, excluding in-cycle and cyclic degradations. Note that in order to be consistent with the properties of the structural elements, the modal capacity curves were also idealized with bilinear curves. P-delta effect was activated in all analyses, and the damping of structures was modeled by modal damping ratio equal to $2 \%$ for each mode.

\subsection{Modal properties}

The roof motion corresponding to the first three natural modes of vibration are shown in Fig. 2Fig. 3 for the 9- and 20-story buildings, respectively.

In the symmetric models (M91 and M201), lateral displacements dominate the motion of the first two modes, while the third mode is purely rotational. Since the third modes of vibration in the symmetric models lack any lateral components, they are not triggered by any of the $\mathrm{x}$ - and $\mathrm{y}$-components of ground accelerations. On the other hand, in asymmetric models, there is a weak coupling between the lateral and torsional components of motion in the third modes, which makes them contribute to the overall response of the buildings.

As M92 and M202 are eccentric only along the x-axis, the first modes of vibration in these two models exhibit torsional rotation coupled with $\mathrm{x}$-lateral motion, and the second modes are dominated by y-lateral motion. Stronger coupling between the lateral and torsional components of motion is observed in the first three modes of M93 and M203, as these models are eccentric along both $\mathrm{x}$ - and $\mathrm{y}$-axes.

In all structural models, the natural vibration period for the dominantly torsional mode (i.e. mode \#3) is shorter than that of the first two modes. This indicates that the buildings are torsionally stiff, which is a quality of structures with lateral load-resisting systems located along their perimeters. In the 9-story buildings, as the plan dimensions and the design of MRFs are identical along the two orthogonal axes, the natural vibration periods of the first two modes are very close, leading to a high correlation factor between the first two modes [Eq. ( 9 )]. In the 20-story buildings, however, the dimensions differ along the $\mathrm{x}$ - and $\mathrm{y}$-axes, so the natural vibration periods of the first two modes are not close. 


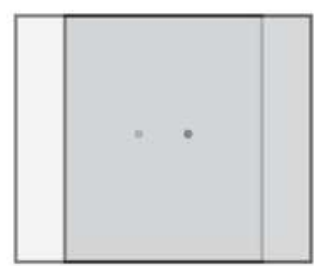

M91)

$\mathrm{T} 1=2.08 \mathrm{~s}$

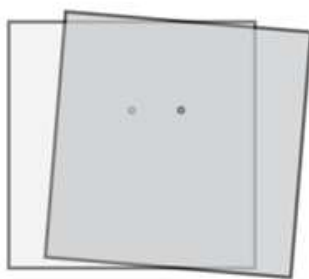

M92)

$\mathrm{T} 1=2.15 \mathrm{~s}$

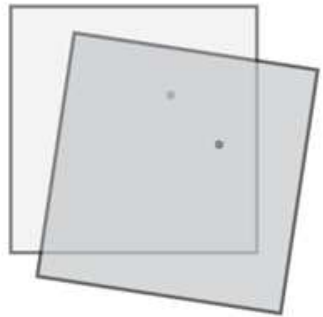

M93) $\mathrm{T} 1=2.22 \mathrm{~s}$

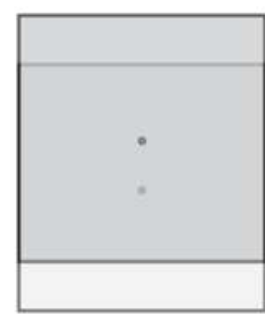

$\mathrm{T} 2=2.08 \mathrm{~s}$

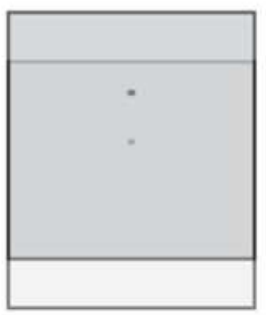

$\mathrm{T} 2=2.08 \mathrm{~s}$

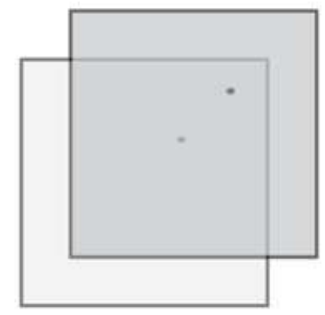

$\mathrm{T} 2=2.08 \mathrm{~s}$

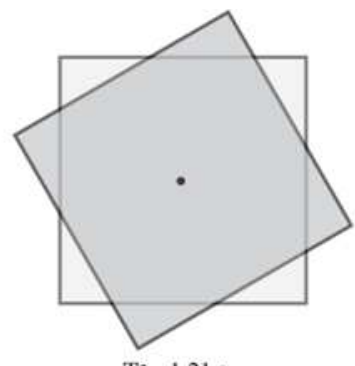

$\mathrm{T} 3=1.21 \mathrm{~s}$

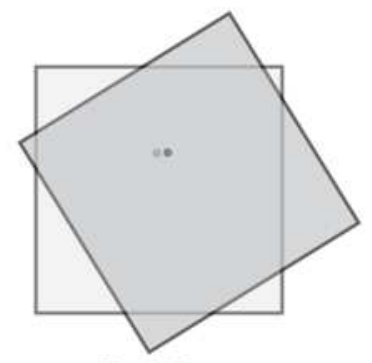

$\mathrm{T} 3=1.17 \mathrm{~s}$

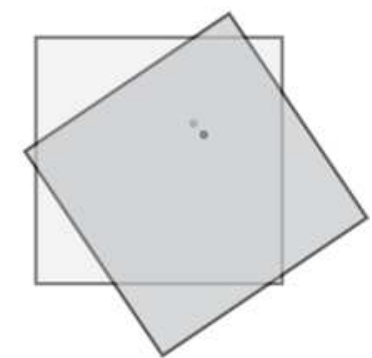

$\mathrm{T} 3=1.14 \mathrm{~s}$

Fig. 2. First triple of periods and modes of vibration for M91, M92, and M93. 


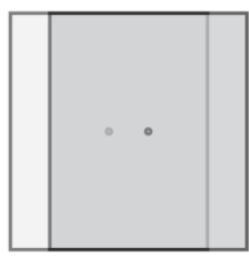

M201)

$\mathrm{T} 1=3.44 \mathrm{~s}$

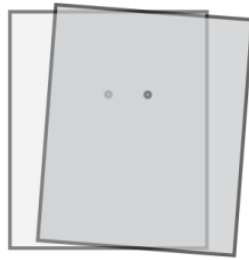

M202)

$\mathrm{T} 1=3.54 \mathrm{~s}$

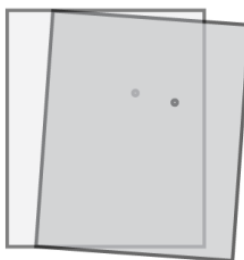

M203)

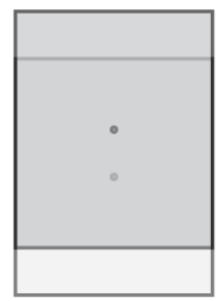

$\mathrm{T} 2=3.12 \mathrm{~s}$

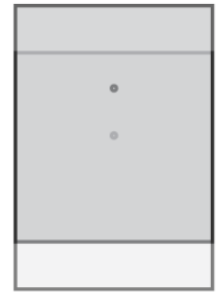

$\mathrm{T} 2=3.12 \mathrm{~s}$

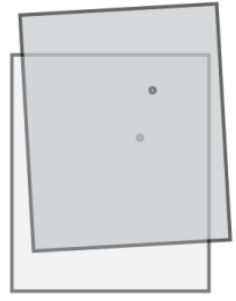

$\mathrm{T} 2=3.18 \mathrm{~s}$

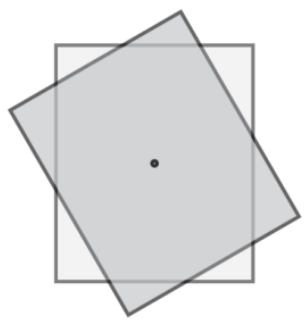

$\mathrm{T} 3=1.81 \mathrm{~s}$

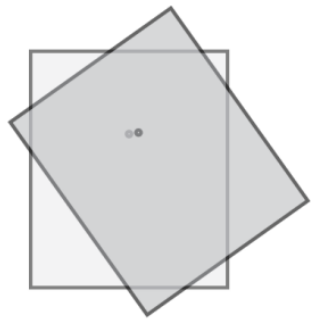

$\mathrm{T} 3=1.77 \mathrm{~s}$

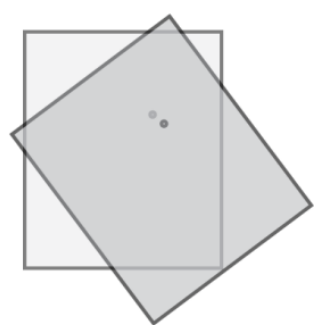

$\mathrm{T} 3=1.72 \mathrm{~s}$

Fig. 3. First triple of periods and modes of vibration for M201, M202, and M203.

\section{Ground motion records}

A set of 22 two-component, far-field ground motion records were considered in this study, adopted from the ground motion database of FEMA-P695 (2009). These ground motions were chosen from 14 different earthquake incidents with magnitudes of at least 6.5. The ground motions were recorded at sites of at least $10 \mathrm{~km}$ away from the fault rupture on soil types $\mathrm{C}$ or $\mathrm{D}$. The peak ground acceleration and velocity of the records are greater than $0.2 \mathrm{~g}$ and 15 $\mathrm{cm} / \mathrm{s}$. The pseudo-acceleration spectra of all ground motion records with their 16th, 50th, and 84th percentile spectra are presented in Fig. 4. For better consistency, each spectrum is normalized to the peak ground acceleration of the xcomponent. 

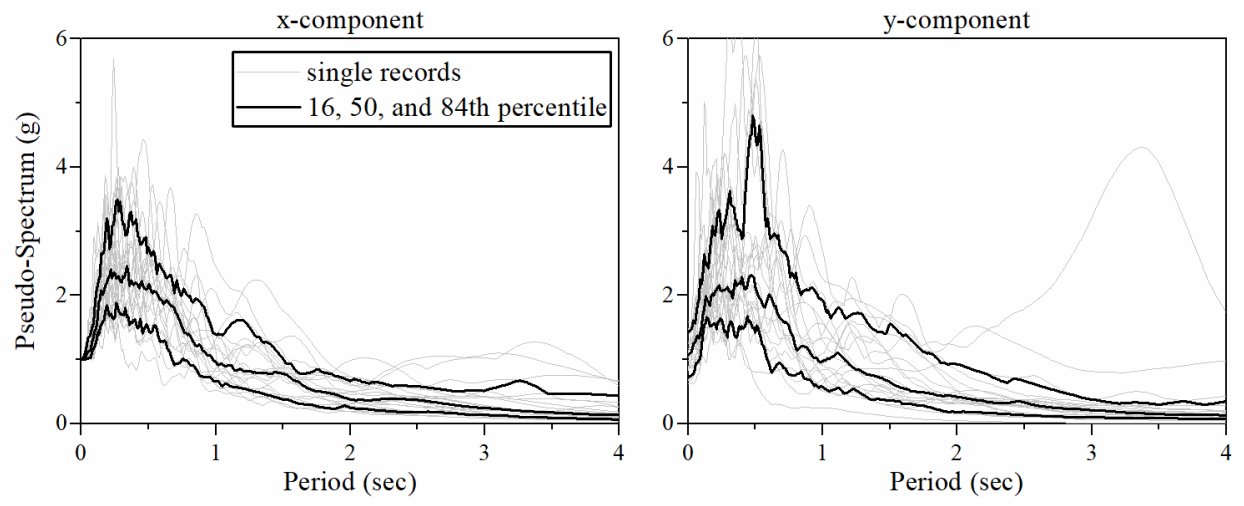

Fig. 4. Pseudo-acceleration spectra for the 22 pairs of ground motion records together with their 16th, 50th, and 84th percentile spectra.

\section{$5 \quad$ Results and discussion}

Next, the results of the exact IDA are compared with those of the BEP. First, the IDA and BEP curves are presented. Then, the accuracy of BEP in estimating the demands at the 'flexible side', CM, and the 'stiff side' is evaluated. The 'flexible side' is defined as the edge of the building closer to the CM, and the 'stiff side' is considered to be the opposite edge. Finally, the median drift-ratios predicted by BEP over the elevations of the buildings are compared with those of the exact NDA for a wide range of ground motion intensity levels.

The preferred choice for intensity measure (IM) is the 5\%-damped pseudo-acceleration spectral ordinate at the firstmode period $\left[S_{a}\left(T_{1}, \zeta_{n}=5 \%\right)\right]$, where the pseudo-acceleration spectrum is considered to be the geometric mean of the pseudo-acceleration spectra associated with the $\mathrm{x}$ - and $\mathrm{y}$-components of ground motions.

To calculate the error in the BEP results, the normalized area between the approximate and the exact IDA curves is taken as an index, which is determined using the following formula:

$$
\operatorname{Error}(\%)=\frac{\int_{0}^{\theta_{\max }(\mathrm{BEP}, \mathrm{IDA})}|\Delta I M| d \theta}{\int_{0}^{\theta_{\max }(\mathrm{IDA})} I M_{\mathrm{IDA}} d \theta} \times 100
$$

In Eq. ( 11 ), $\theta$ is the maximum of the peak inter-story drift-ratios, extracted from the whole or a part of the structure under assessment. The term $I M_{I D A}$ is the ground acceleration intensity for the IDA curves, and $\Delta I M$ represents the difference between the IMs of BEP and IDA for a given drift ratio. The term $\theta_{\max }$ (IDA) is the maximum drift ratio of the IDA curve (i.e., the drift ratio at which the curve flattens), and $\theta_{\max }(\mathrm{BEP}, \mathrm{IDA})$ is the maximum drift ratio considering both BEP and IDA curves.

Unlike the displacement-based methods which often encounter distortions in the pushover curves of higher modescommonly seen as reversals in capacity curves (Hernandez-Montes, Kwon et al. 2004, Soleimani, Aziminejad et al. 2018) - BEB showed to have no limitation for the inclusion of higher modes. The minimum number of modes to 
achieve maximum accuracy was found to be 9 and 12 modes for the 9- and 20-story buildings, respectively; including more modes did not show much influence on the final results. To examine the effect of higher modes on the overall response, the results derived from the first triple modes are also reported. The discussions in this section are based on the results obtained from the 9- and 12-mode BEP (for 9- and 12-story models), unless otherwise stated.

\subsection{IDA curves}

Shown in Fig. 5 Fig. 6 are the 16th, 50th, and 84th percentile IDA curves together with the predicted BEP curves for the 9 - and 20-story buildings, respectively.

An important observation on Fig. 5 Fig. 6 is that BEP offers similar accuracies for both symmetric and asymmetric models. In the 9-story buildings, for example, BEP predicted the medium IDA curve of the symmetric model (M91) with 9\% error, and the errors for M92 and M93 were 6\% and 10\%, respectively. Similarly, the median IDA curves for the 20-story buildings were predicted with roughly the same degree of accuracy $(15 \%, 15 \%$, and $14 \%$ error for M201, M202, and M203, respectively). It shows that the accuracy of the method was not deteriorated by the presence of asymmetry in structures.

The results also demonstrate that BEP provides slightly lower accuracy in the 20-story buildings compared to the 9story buildings. Considering both symmetric and asymmetric models, the average error of the median IDA curves was $8.3 \%$ for the 9 -story buildings and $16.7 \%$ for the 20 -story buildings. This can be compared to the results of the previous study on a 3-story, asymmetric structure subjected to the same ensemble of ground motion records, in which the error of the median BEP curves was lower than $4 \%$. The observation allows for the conclusion that the accuracy of BEP decreases slightly with the increase in the height of the structures.

As a general tendency in both 9- and 20-story buildings, the 16th percentile curves were estimated with the highest accuracy, followed by the 50th and 84th percentile curves, respectively. Taking all structural models into account, the mean error of BEP in predicting the 16th percentile curves was equal to $5.6 \%$, compared to $11.5 \%$ and $15.7 \%$ for the 50th and 84th percentile curves, respectively. The only exception to this trend was M93, for which $10 \%$ error was reported for all three percentile BEP curves. The most significant error among all BEP curves was observed in the 84th percentile curve for M91, in which a surge of the IDA curve at around 3.5\% drift-ratio was not detected by BEP.

A comparison of the results between the 9-mode and 3-mode BEP in Fig. 5 demonstrates that the first three modes are not adequate to predict IDA curves of the 9-story buildings. This is in contrast to the results of previous research on low-rise buildings, for which the first triple modes were sufficient to acquire reasonable accuracy (Soleimani, Aziminejad et al. 2018). Considering the median IDA curves as a criterion, the average error for the 9-mode BEP was equal to $8.3 \%$, compared to $22.3 \%$ for the 3 -mode BEP. Generally, the 3-mode BEP tends to overestimate the intensity of ground acceleration for a given drift- ratio demand. 
As one might expect, this trend is more noticeable in high-rise buildings (Fig. 6). Considering all 20-story models, 12-mode BEP estimated the median IDA curves with an average error of $14.7 \%$, while that of the 3-mode BEP was equal to $39.3 \%$. It should be noted that the maximum drift-ratios determined by the 3-mode BEP are not always extracted from the same stories that the maximum drift-ratios take place in IDA. The first three modes predominately cause drift-ratio demands in lower stories, while the maximum drift ratio in medium and high-rise buildings often takes place in upper stories, mostly caused by higher modes (Fig. 9- Fig. 12).

Regarding computational effort, BEP roughly takes $3 \%$ of the computer time required for the exact IDA. In our analyses, using a Core i7, $2.9 \mathrm{GHz}$ processor, around 6 hours were needed to perform IDAs for each 9-story building considering all the 22 ground motion records, and this time was around 9 hours for the 20 -story buildings. On the other hand, BEP took only 11 and 15 minutes to produce approximate IDA curves for the 9- and 20-story buildings, respectively. In case only the first three modes were included, the computing time for BEP was about 4 minutes for each building. 

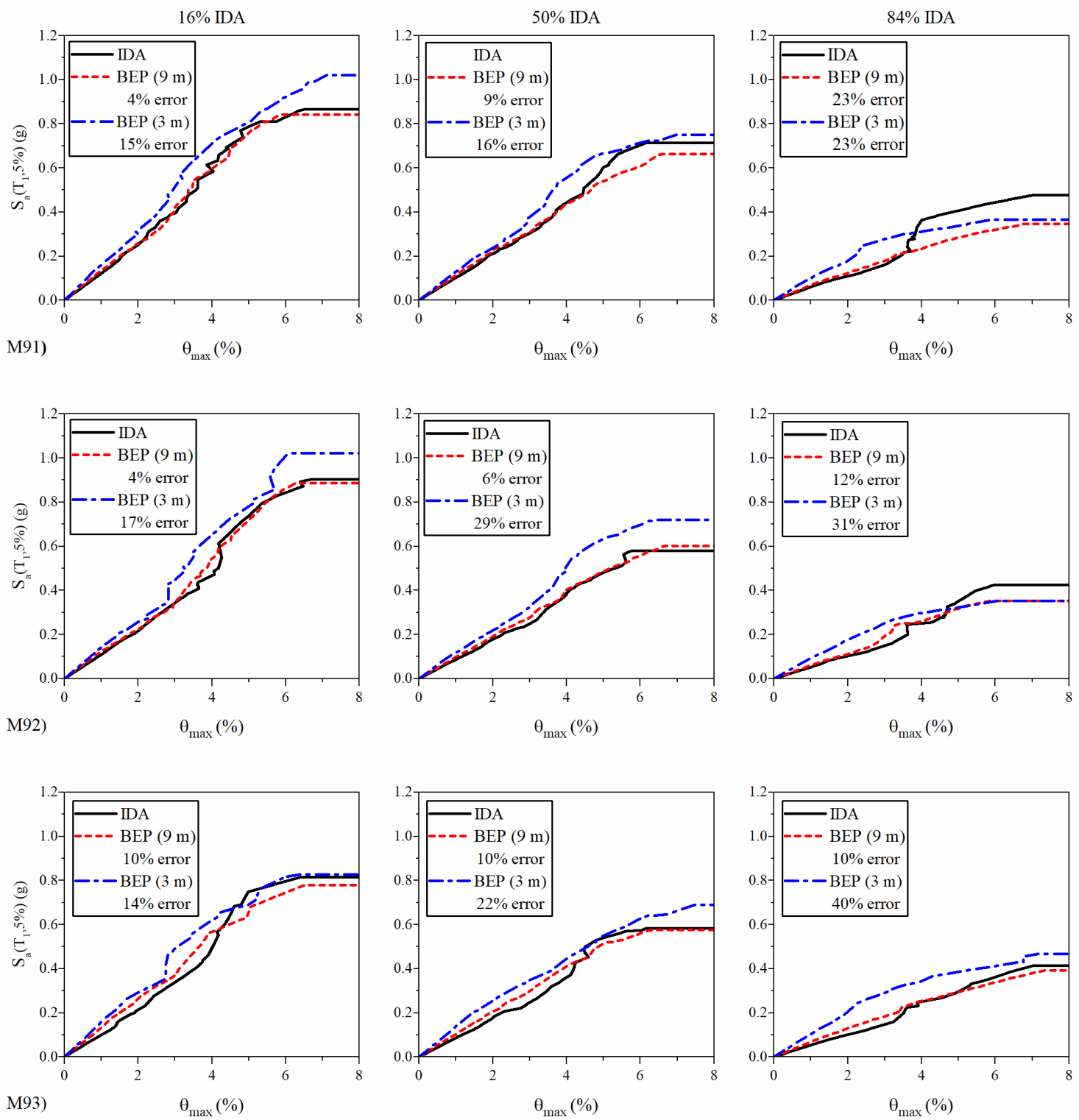

Fig. 5. Sixteenth, 50th, and 84th percentile IDA curves together with the 3-and 9-mode BEP curves for M91, M92, and M93. The x- and y-axes represent the maximum drift-ratio and IM, respectively. 

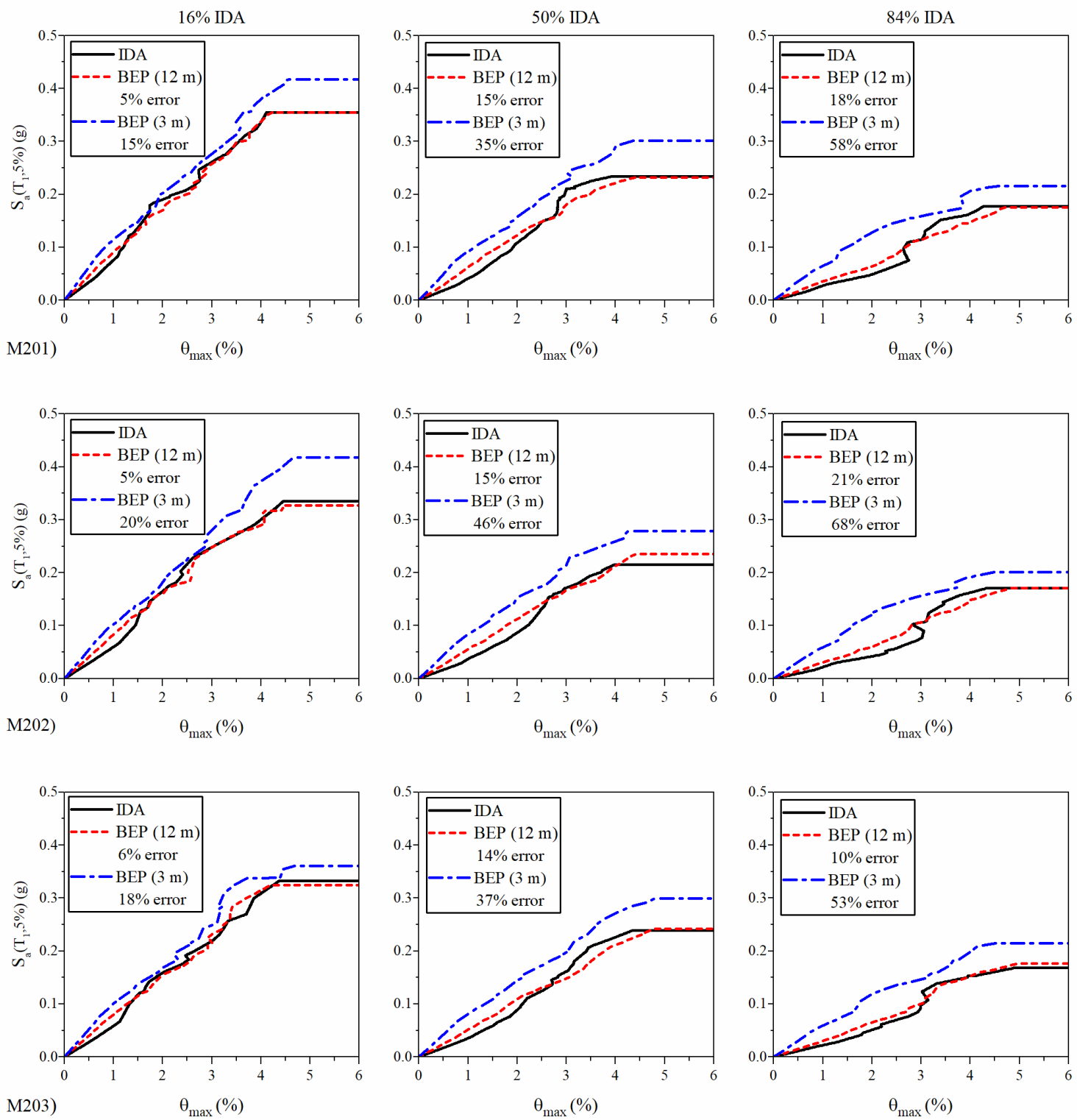

Fig. 6. Sixteenth, 50th, and 84th percentile IDA curves together with the 3- and 12-mode BEP curves for M201, M202, and M203. The x- and yaxes represent the maximum drift-ratio and IM, respectively.

\subsection{Stiff and flexible sides}

The IDA curves presented in the previous section reflect the maximum of the peak drift-ratios throughout the whole structure. In asymmetric buildings, these maximum drift ratios often take place at the 'flexible side' of the buildings. To examine the accuracy of BEP in predicting the demands over the plan of the asymmetric models, the BEP and IDA curves for the 'flexible side', CM, and the 'stiff side' are separately presented in this section. The 'flexible side' comprises the northern and eastern MRFs as they are the closest to the CM, and the 'stiff side' consists of the 
southern and western MRFs. The drift ratios at CMs are also provided for the sake of comparison, which encompass the demands along both $\mathrm{x}$ - or $\mathrm{y}$-directions.

The results for M93 and M203 are presented in Fig. 7 Fig. 8, respectively. It is found that the accuracy of the method remains consistent over the plan of the asymmetric buildings, with no systematic bias observed. For M93 (Fig. 7), the average errors of the BEP curves at the 'flexible side', CM, and 'stiff side' were 10\%, 8\%, and 13\%, respectively. The trend was similar in M203, with 12\%, 13\%, and 9\% errors for the 'flexible side', CM, and the 'stiff side', respectively. The results of M92 and M202 are not reported for the sake of brevity, but the findings apply to those models as well.
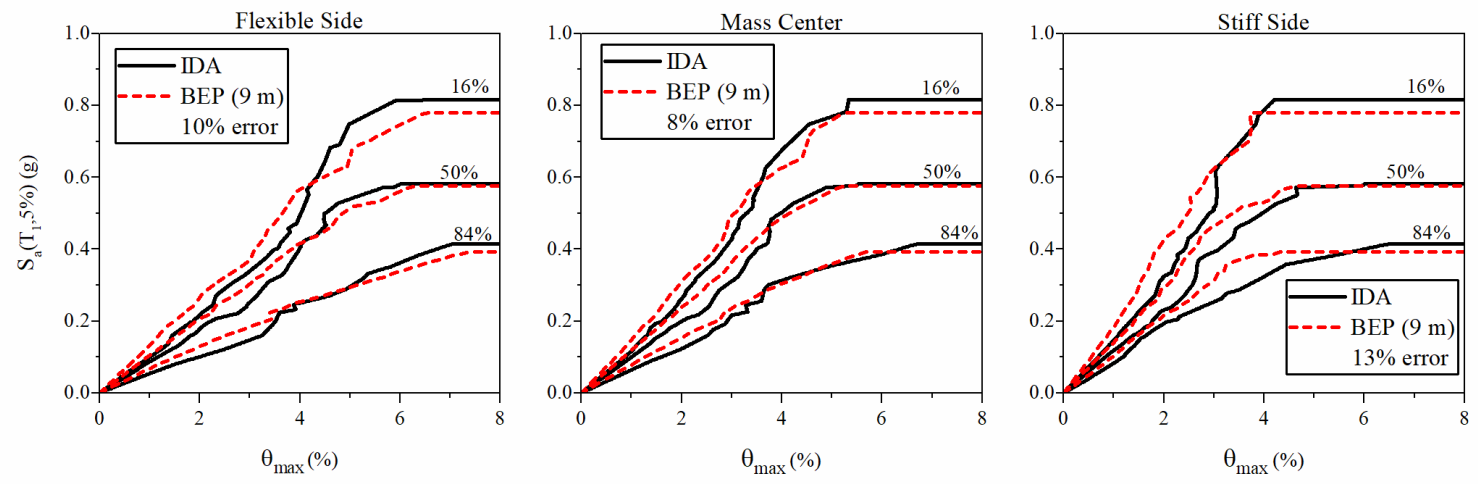

Fig. 7. Sixteenth, 50th, and 84th percentile IDA curves together with the BEP curves at the 'flexible side', mass center, and 'stiff side' for M93.
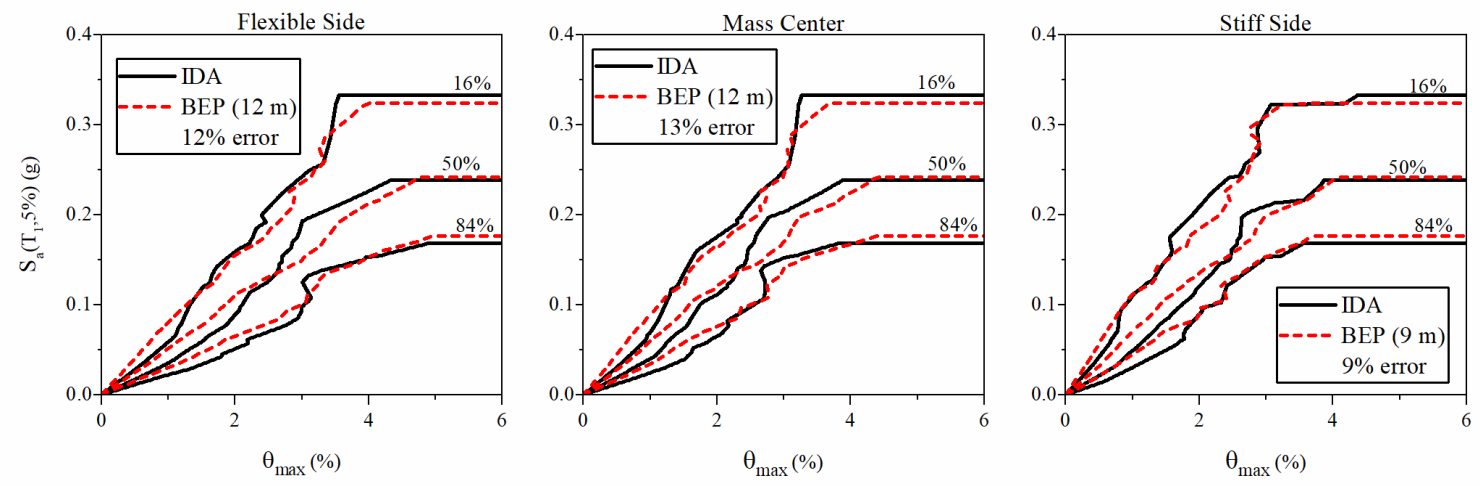

Fig. 8. Sixteenth, 50th, and 84th percentile IDA curves together with the BEP curves at the 'flexible side', mass center, and 'stiff side' for M203. 


\subsection{Drift ratios over the elevations}

To examine the accuracy of the demands over the height of the buildings, the BEP and NDA median drift-ratios over the elevations are compared in Fig. 9- Fig. 12. The chosen MRFs for this purpose are those at the northern edge (oriented in the $\mathrm{x}$-direction) and the eastern edge (oriented in the y-direction) because, being located at the 'flexible edge,' these frames are more likely to experience the maximum demands.

Fig. 9 andFig. 10 show the median drift-ratio demands of the northern and eastern MRFs, respectively, for the 9story buildings. Four ground motion intensities are covered, ranging from $\mathrm{S}_{a}\left(T_{1}\right)=0.1 \mathrm{~g}$ to $0.5 \mathrm{~g}$. Those of the 20story buildings are also presented in Fig. 11 andFig. 12, covering four ground motion intensities varying from $\mathrm{S}_{a}\left(T_{1}\right)=0.1 \mathrm{~g}$ to $0.4 \mathrm{~g}$. For each diagram, the height-wise average error of the BEP drift-ratios is also reported. The following formula is used to calculate the errors:

$$
\operatorname{Error}(\%)=\frac{\sum_{1}^{N}\left|\theta_{\mathrm{BEP}}-\theta_{\mathrm{NDA}}\right|}{\sum_{1}^{N} \theta_{\mathrm{NDA}}} \times 100
$$

where $\theta_{\mathrm{BEP}}$ and $\theta_{\mathrm{NDA}}$ are the median drift-ratio demands for BEP and NDA, and $N$ is the number of stories.

As is demonstrated in Fig. 9- Fig. 12, when higher modes were included, the median drift-ratios predicted by BEP were reasonably consistent with the results of NDA. The range of errors lied between $4 \%$ and $20 \%$. The average errors, considering all the diagrams, were equal to $9 \%$ and $12 \%$ for the 9 - and 20 -story buildings, respectively. The highest error among the 9-story buildings was $19 \%$, observed in M93 at $\mathrm{S}_{a}\left(T_{1}\right)=0.35 g$. For the 20-story buildings, the largest error was $20 \%$ in almost all models at $\mathrm{S}_{a}\left(T_{1}\right)=0.1 \mathrm{~g}$.

A tendency is observed in the 20-story buildings for BEP to underestimate the drift-ratio demands in the elastic domain (i.e., $\mathrm{S}_{a}\left(T_{1}\right)=0.1 \mathrm{~g}$ ). This bias can also be seen in the IDA curves of all 20-story models (Fig. 6), as the estimated IDA curves lie marginally over the exact IDA curves throughout the linear range of the building behavior. This is interpreted to be due to an error in the combination of modal responses by the modified CQC formula, since this is the only approximation included in BEP when the structure is still elastic. As this trend is observed in both symmetric and asymmetric models, the error cannot be related to the modifications made to the CQC formula; note that the modified CQC will be equivalent to the original CQC in the symmetric buildings. Moreover, in the elastic phase of the symmetric models, the BEP procedure will be equivalent to the well-known response spectrum analysis (RSA), and the CQC method used in RSA is known to estimate the peak responses generally on the unconservative side, with errors up to 25\% being reported in previous studies [Chapter 13-(Chopra 2011)]. This is in agreement with the results of this research. Nevertheless, these errors were only observed in the 20-story buildings, and the median drift-ratios over the elastic range of the 9-story buildings were predicted quite accurately. Furthermore, for the 20-story buildings at higher intensities (i.e., $S_{a}\left(T_{1}\right)=0.2 g, 0.3 g$ and $0.4 g$ ), which drive the structures into the 
nonlinear range, BEP has provided more accurate estimates of the median peak responses compared to that observed in the linear range (Fig. 6).

Over the first five stories of the 9-story buildings (Fig. 9Fig. 10), little difference is observed between the drift-ratio demands of the 3-and 9-mode BEP, which implies that higher modes have a minimal share in the drift-ratio demands of the lower stories (the first five stories). On the other hand, higher modes are inferred to have a substantial contribution to the drift-ratio demands of the upper stories, as there are large discrepancies between the results of the 3- and 9-mode BEP in the upper stories. In 20-story buildings, this trend changes to only a limited degree. Higher modes appear to have relatively more contribution to the drift-ratio demands of lower stories in the 20-story models (the first 10 stories), but the demands at the upper stories remain largely linked to the higher modes (Fig. 11 Fig. 12).

No significant alteration in the BEP accuracy was detected throughout the nonlinear range of structural behavior (Fig. 9- Fig. 12). Nevertheless, there is a tendency to underestimate the drift ratios at the upper stories unless in the most intense ground motion levels. For almost all of the buildings considered, the median drift ratios of the upper stories were underestimated in the intensities $\mathrm{S}_{a}\left(T_{1}\right)=0.1 \mathrm{~g}$ and $0.2 \mathrm{~g}$, but they were more accurately estimated in the higher intensities, albeit with some exceptions among the 20-story models.

Comparing the median drift-ratios in the northern and eastern MRFs (Fig. 9 Fig. 11 compared to Fig. 10 Fig. 12 ) shows a consistency in the accuracy of the results between the $\mathrm{x}$ - and $\mathrm{y}$-directions. Considering all four intensity levels, the average drift-ratio error in the northern MRF of the 9-story buildings was equal to 10.2\% (Fig. 9), compared to $8 \%$ for the eastern MRF (Fig. 10). For 20-story buildings, the average errors in both edges were similar at around $12 \%$ (Fig. 11Fig. 12). 

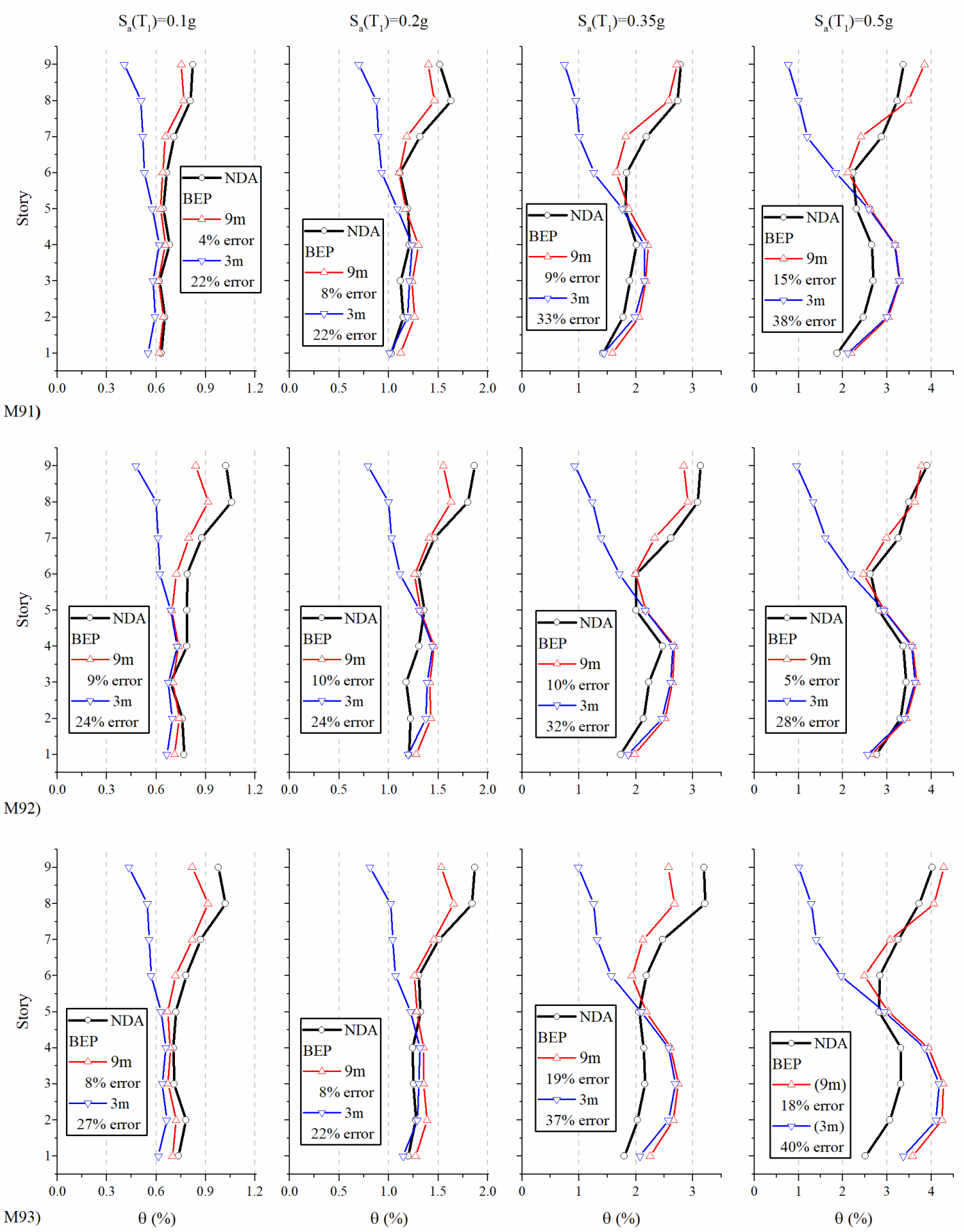

Fig. 9. Median drift-ratios by NDA and BEP (3- and 9-mode) at the northern MRF of M91, M92, and M93 for the ground motion intensities $\mathrm{S}_{\mathrm{a}}\left(T_{1}\right)=0.1 g, 0.2 g, 0.35 g$, and $0.5 g$. 

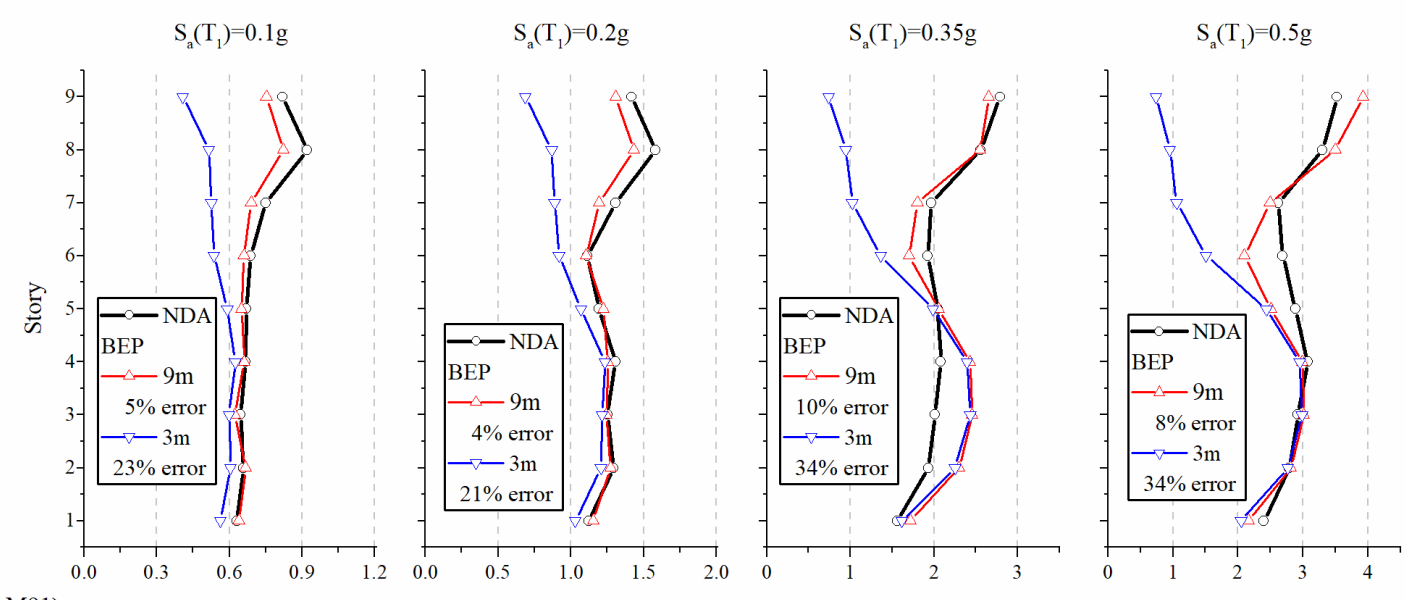

M91)
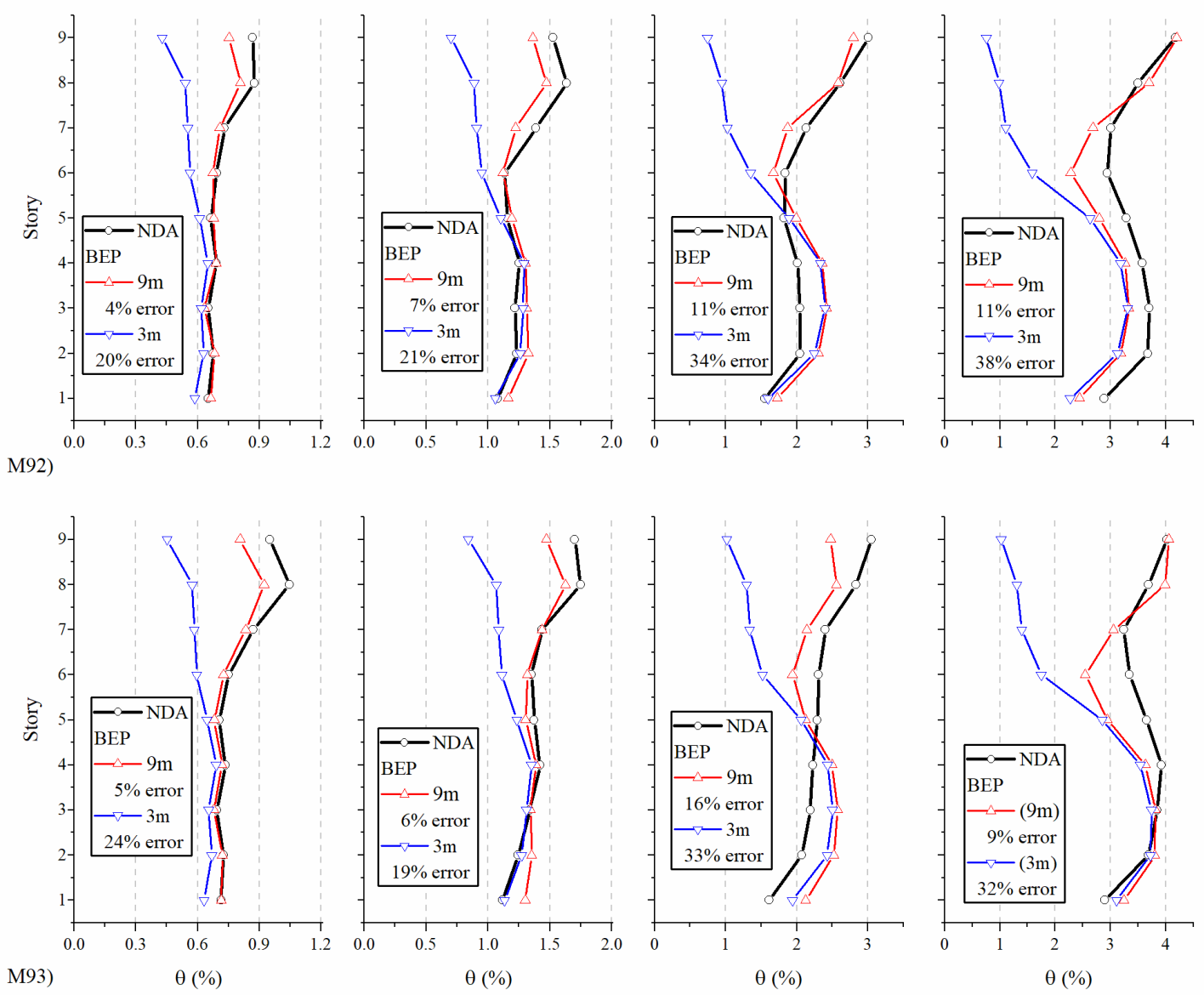

Fig. 10. Median drift-ratios by NDA and BEP (3- and 9-mode) at the eastern MRF of M91, M92, and M93 for the ground motion intensities $\mathrm{S}_{\mathrm{a}}\left(T_{1}\right)=0.1 g, 0.2 g, 0.35 g$, and $0.5 g$. 

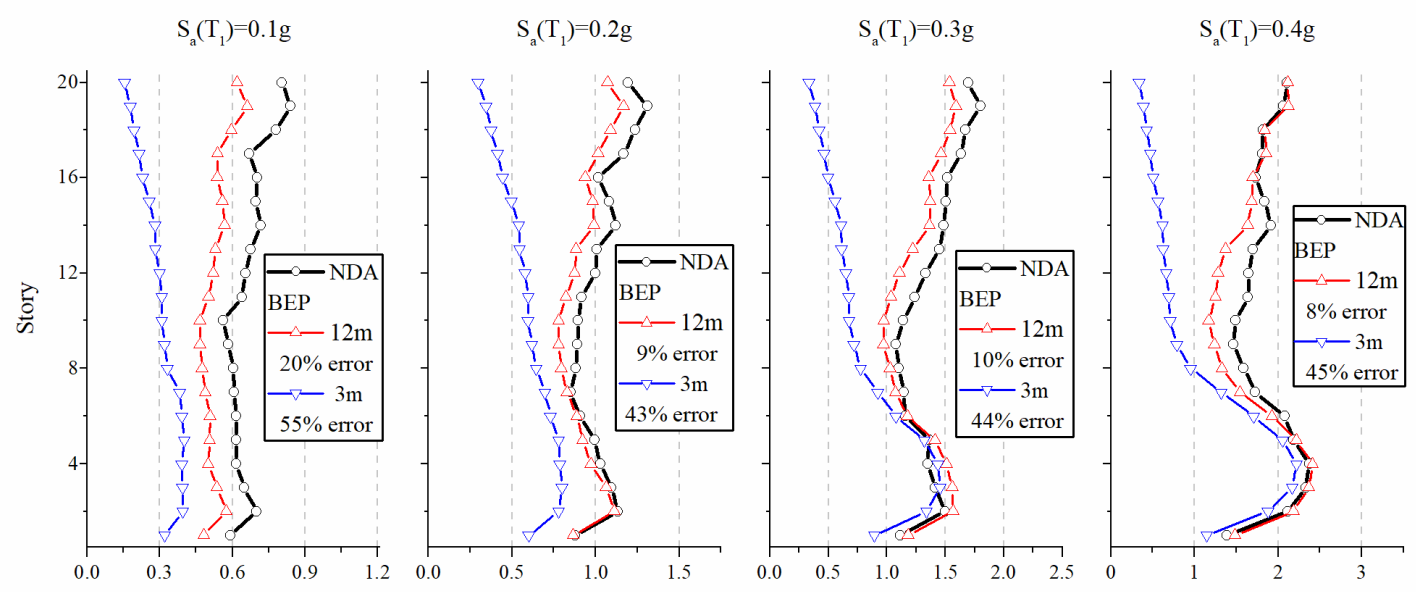

M201)
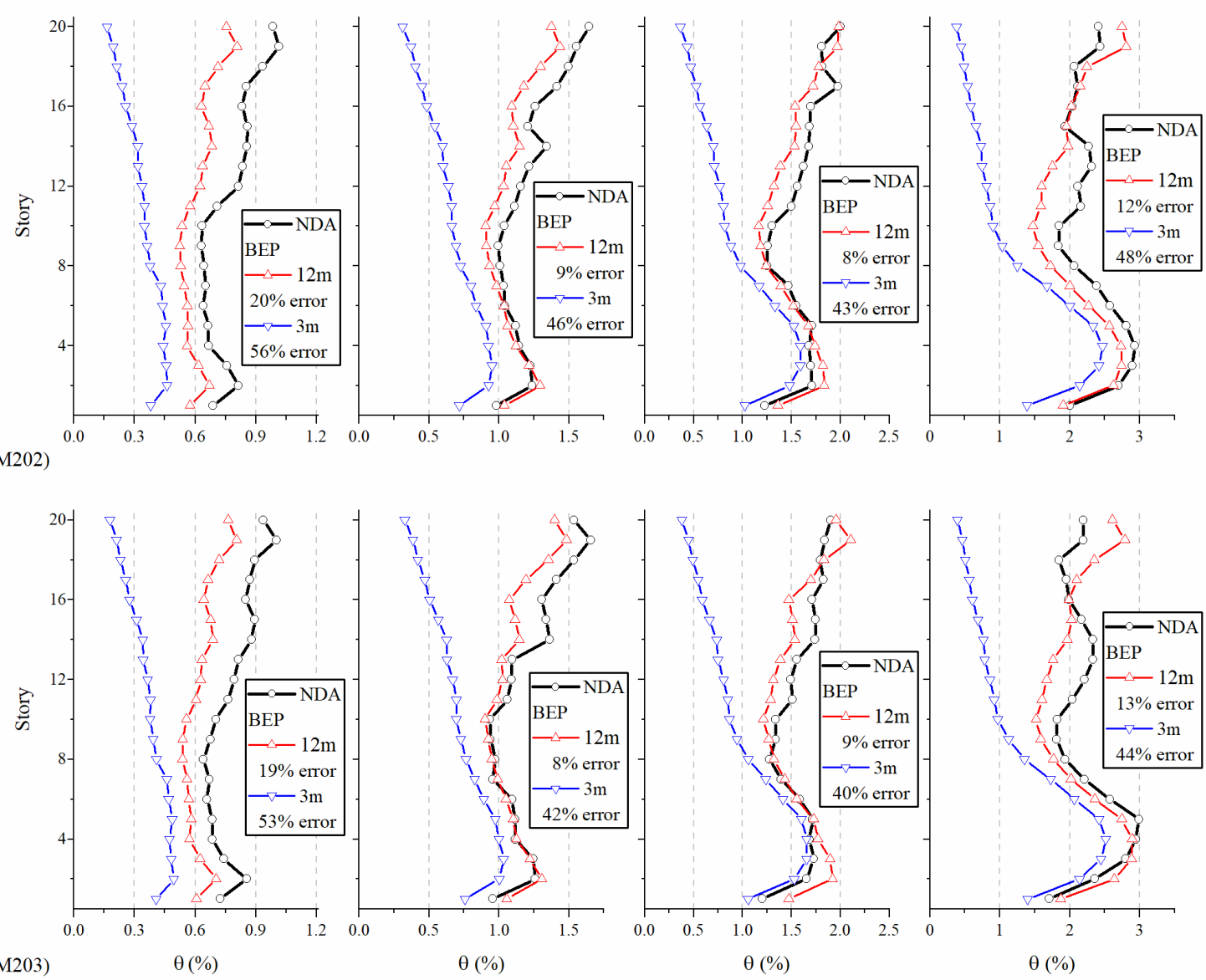

Fig. 11. Median drift-ratios by NDA and BEP (3- and 12-mode) at the northern MRF of M201, M202, and M203 for the ground motion intensities $\mathrm{S}_{\mathrm{a}}\left(T_{1}\right)=0.1 \mathrm{~g}, 0.2 \mathrm{~g}, 0.3 \mathrm{~g}$, and $0.4 \mathrm{~g}$. 

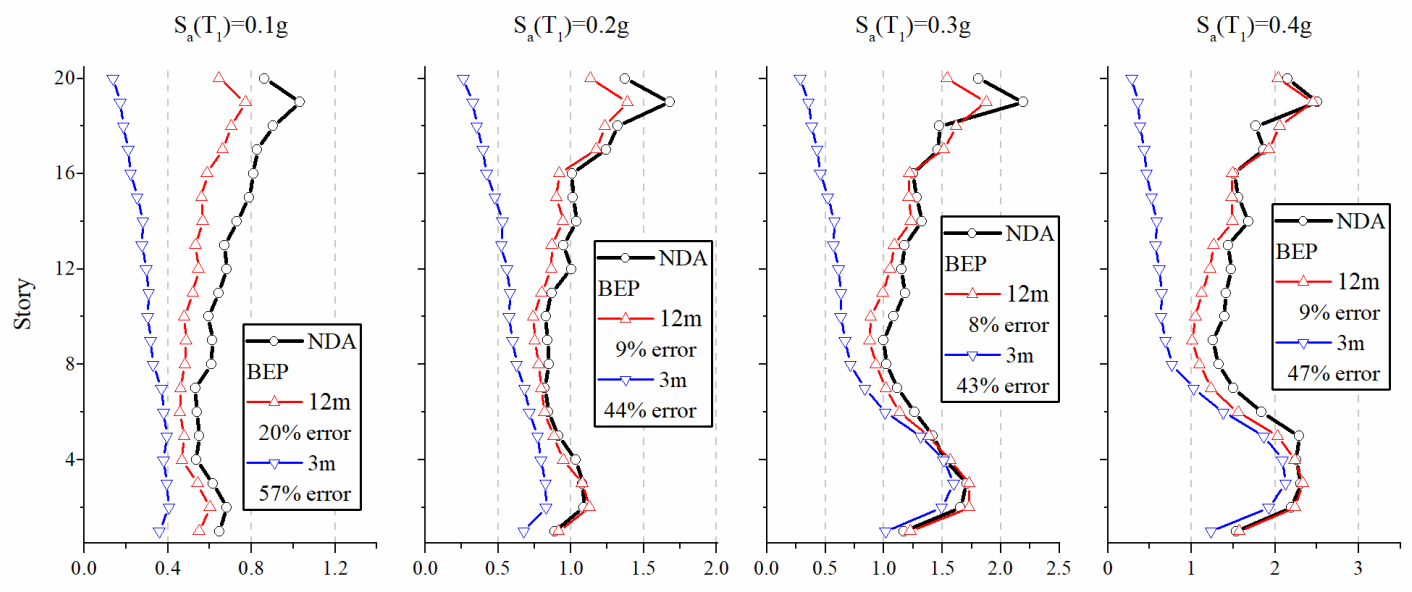

M201)
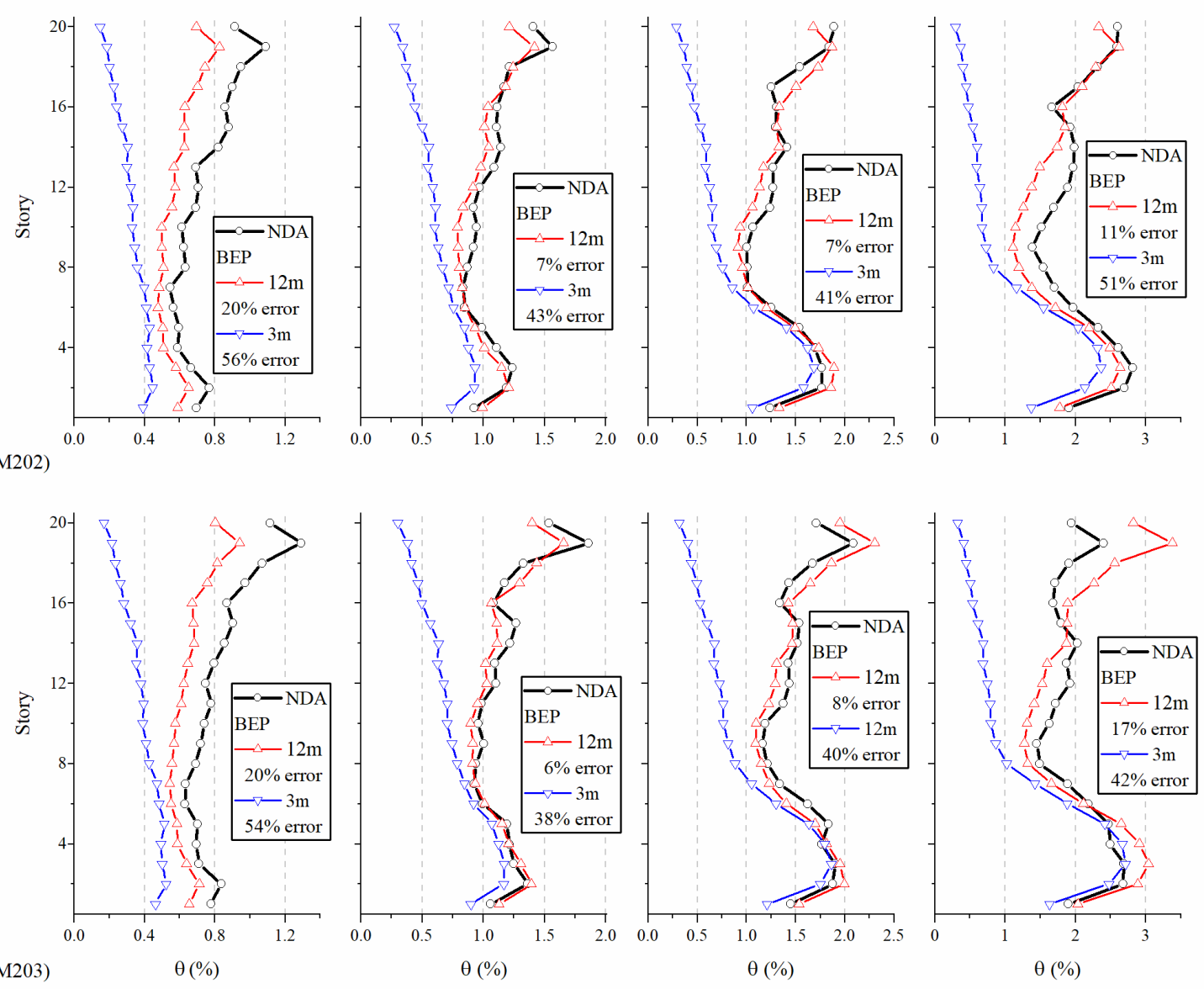

Fig. 12. Median drift-ratios by NDA and BEP (3- and 12-mode) at the eastern MRF of M201, M202, and M203 for the ground motion intensities $\mathrm{S}_{\mathrm{a}}\left(T_{1}\right)=0.1 g, 0.2 \mathrm{~g}, 0.3 g$, and $0.4 g$. 


\section{Conclusion}

BEP, which has already proved to be useful in low-rise buildings, is evaluated for medium- and high-rise buildings. The accuracy of the method to predict two-component IDA curves is assessed as well as its utility to estimate median drift-ratios over the height of the structures. Six structural models were considered for this evaluation in two groups of 9- and 20-story buildings. Each group consisted of one symmetric model, one asymmetric model about the $\mathrm{y}$-axis, and one asymmetric model about both $\mathrm{x}$ - and $\mathrm{y}$-axes. The assessment was carried out under an ensemble of 22 two-component ground motion records, and the results led to the following conclusions:

- BEP provides sufficiently accurate IDA curves for medium- and high-rise buildings under biaxial seismic excitations, while it takes only $3 \%$ of the computational time required for the exact IDA.

- Having a significant share in the dynamic response of medium- and high-rise buildings, higher mode effects are well detected in BEP.

- The accuracy of the method is not affected by asymmetry in buildings, i.e., the method offers the same degree of accuracy in asymmetric buildings as it does in symmetric buildings.

- The accuracy of BEP slightly decreases with the increase in the height of buildings. Previous research showed around $4 \%$ error in predicting the median IDA curves of low-rise buildings, while this figure increased to $8.3 \%$ and $16.7 \%$ for medium- and high-rise buildings, respectively, as this study revealed.

- The 16th percentile IDA curves are predicted with the highest accuracy (5.6\% average error), followed by 50 th and 84 th percentile curves, respectively ( $11.5 \%$ and $15.7 \%$ average error).

- The drift ratios at the 'flexible side,' CM, and the 'stiff side' of asymmetric buildings are determined with roughly the same accuracy level.

- $\quad$ BEP estimates the median inter-story drift-ratios reasonably accurate over the height of the medium and highrise structures.

\section{References}

Azarbakht, A. and M. Dolšek (2007). "Prediction of the median IDA curve by employing a limited number of ground motion records." Earthquake Engineering \& Structural Dynamics 36(15): 2401-2421.

Baltzopoulos, G., R. Baraschino, I. Iervolino and D. Vamvatsikos (2017). "SPO2FRAG: software for seismic fragility assessment based on static pushover." Bulletin of Earthquake Engineering 15(10): 4399-4425.

Birzhandi, M. S. and A. M. Halabian (2017). "Application of 2DMPA method in develpoing fragility curves of planasymmetric structures." Engineering Structures 153: 540-549.

Birzhandi, M. S. and A. M. Halabian (2018). "A new simplified approach for assessing nonlinear seismic response of plan-asymmetric structures considering soil-structure interaction." Bulletin of Earthquake Engineering 16(12): 6013-6046.

Birzhandi, M. S. and A. M. Halabian (2020). "Fast fragility analysis of plan-asymmetric structures considering soilstructure interaction using flexible base 2 degrees of freedom Modal Pushover Analysis (F2MPA)." Soil Dynamics and Earthquake Engineering 138: 106270. 
Brozovič, M. and M. Dolšek (2014). "Envelope-based pushover analysis procedure for the approximate seismic response analysis of buildings." Earthquake Engineering \& Structural Dynamics 43(1): 77-96.

Chopra, A. K. (2011). "Dynamics of structures: theory and applications to earthquake engineering." Prentice-Hall international series in civil engineering and engineering mechanics.

Chopra, A. K. and R. K. Goel (2002). "A modal pushover analysis procedure for estimating seismic demands for buildings." Earthquake Engineering \& Structural Dynamics 31(3): 561-582.

CSI (2011). PERFORM 3D . User Guide v5, Non-linear Analysis and Performance Assessment for 3D Structures, Computers and Structures, Inc. Berkeley, CA.

Dolšek, M. and P. Fajfar (2007). "Simplified probabilistic seismic performance assessment of plan-asymmetric buildings." Earthquake engineering \& structural dynamics 36(13): 2021-2041.

FEMA (2005). Improvement of nonlinear static seismic analysis procedures. FEMA-440, Redwood City, Federal Emergency Management Agency.

FEMA (2009). Quantification of Building Seismic Performance Factors. FEMA P695, Washington, DC, Federal Emergency Management Agency.

Gupta, A. and H. Krawinkler (1999). Seismic demands for the performance evaluation of steel moment resisting frame structures, Stanford University Stanford.

Han, S. W. and A. K. Chopra (2006). "Approximate incremental dynamic analysis using the modal pushover analysis procedure." Earthquake Engineering \& Structural Dynamics 35(15): 1853-1873.

Hernandez-Montes, E., O.-S. Kwon and M. A. Aschheim (2004). "An energy-based formulation for first-and multiple-mode nonlinear static (pushover) analyses." Journal of Earthquake Engineering 8(1): 69-88.

Lin, J. L. and K. C. Tsai (2007). "Simplified seismic analysis of asymmetric building systems." Earthquake Engineering \& structural dynamics 36(4): 459-479.

Peruš, I., R. Klinc, M. Dolenc and M. Dolšek (2013). "A web-based methodology for the prediction of approximate IDA curves." Earthquake Engineering \& Structural Dynamics 42(1): 43-60.

Saedi-Daryan, A., S. Soleimani and M. Hasanzadeh (2018). "Extension of the Modal Pushover Analysis to Assess Structures Exposed to Blast Load." Journal of Engineering Mechanics 144(3): 04018006.

Saedi Daryan, A., S. Soleimani and H. Ketabdari (2017). "A modal nonlinear static analysis method for assessment of structures under blast loading." Journal of Vibration and Control: 1077546317708517.

Soleimani, S., A. Aziminejad and A. Moghadam (2017). "Extending the concept of energy-based pushover analysis to assess seismic demands of asymmetric-plan buildings." Soil Dynamics and Earthquake Engineering 93: $29-41$.

Soleimani, S., A. Aziminejad and A. Moghadam (2018). "Approximate two-component incremental dynamic analysis using a bidirectional energy-based pushover procedure." Engineering Structures 157: 86-95.

Vamvatsikos, D. and C. A. Cornell (2002). "Incremental dynamic analysis." Earthquake Engineering \& Structural Dynamics 31(3): 491-514.

Vamvatsikos, D. and C. A. Cornell (2005). "Direct estimation of seismic demand and capacity of multidegree-offreedom systems through incremental dynamic analysis of single degree of freedom approximation 1." Journal of Structural Engineering 131(4): 589-599.

Zarfam, P. and M. Mofid (2011). "On the modal incremental dynamic analysis of reinforced concrete structures, using a trilinear idealization model." Engineering Structures 33(4): 1117-1122. 

Figures

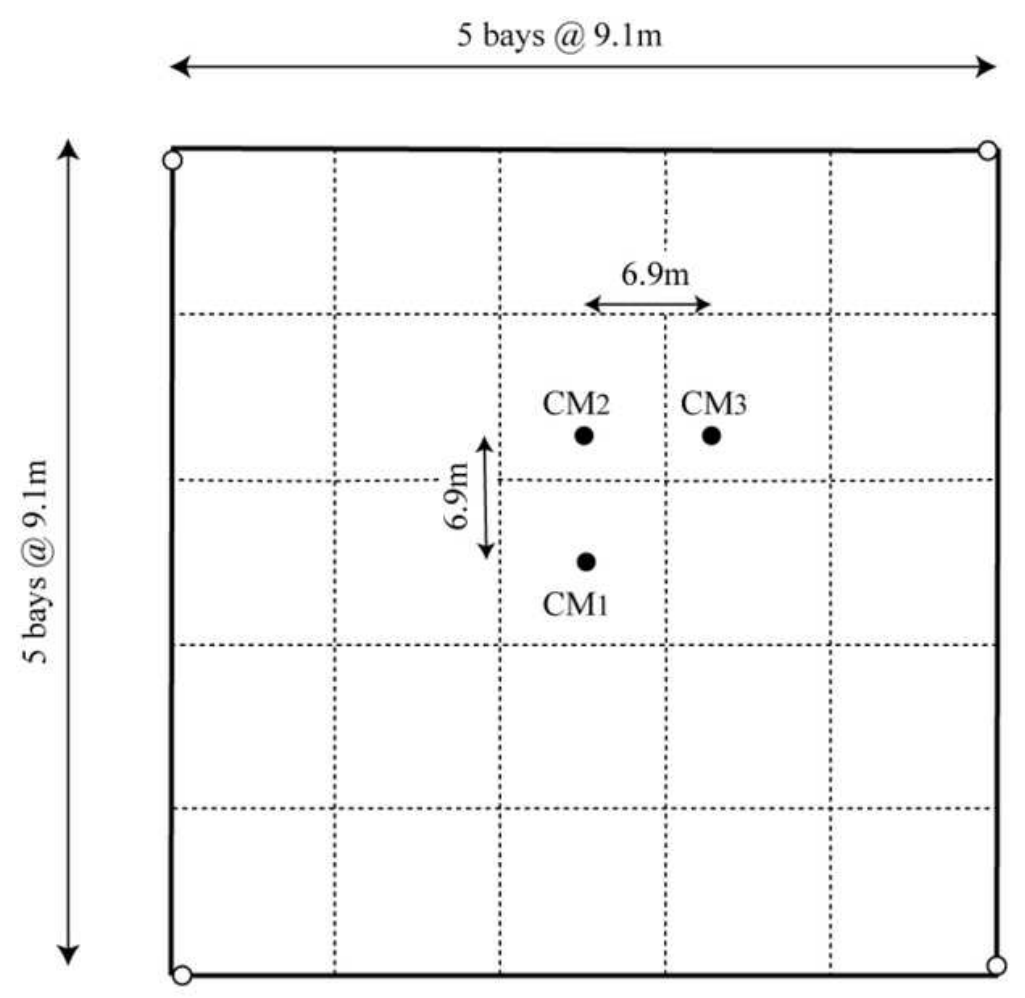

a)

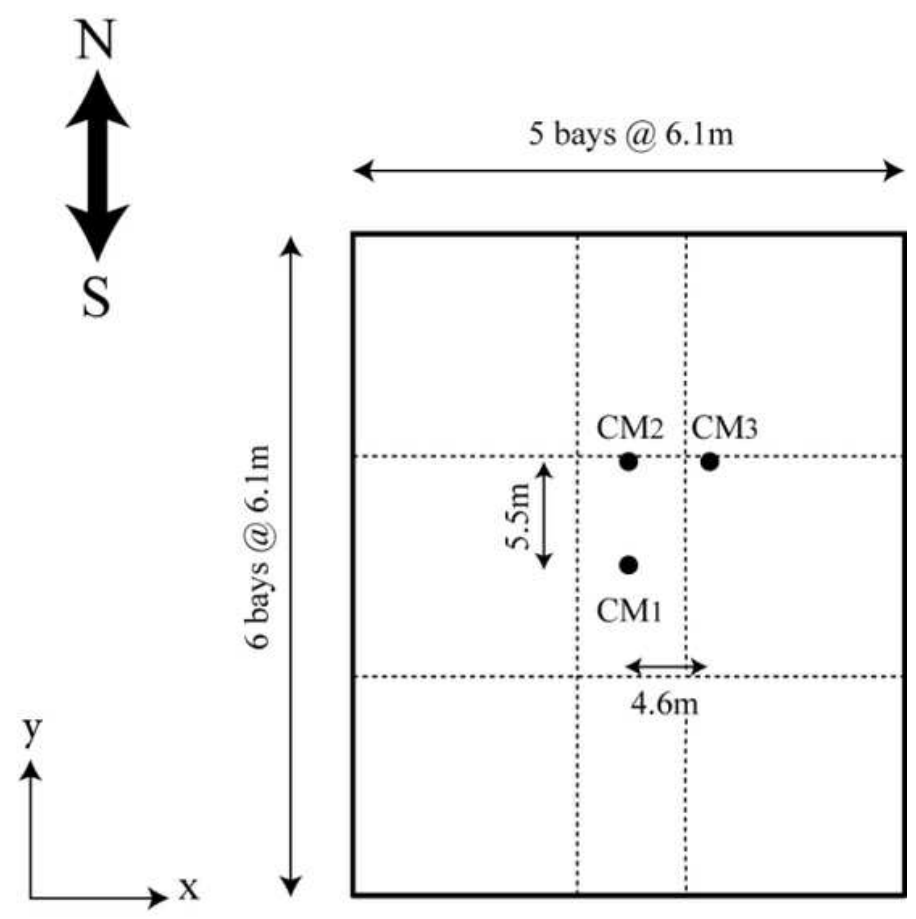

b)

\section{Figure 1}

The plans of a) 9-story and b) 20-story structural models. The points indicated by CM1, CM2 and CM3 are the mass centers of different models in each category. Solid lines represent moment resisting frames. 


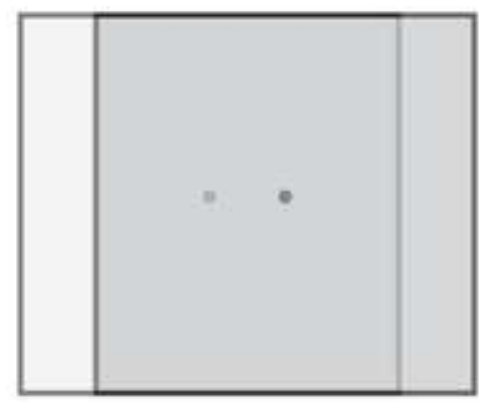

M91) $\quad \mathrm{T} 1=2.08 \mathrm{~s}$

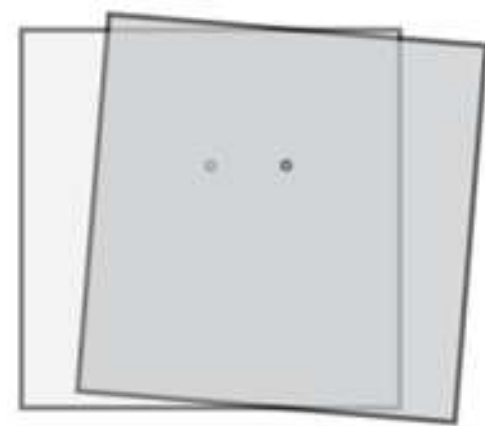

M92)

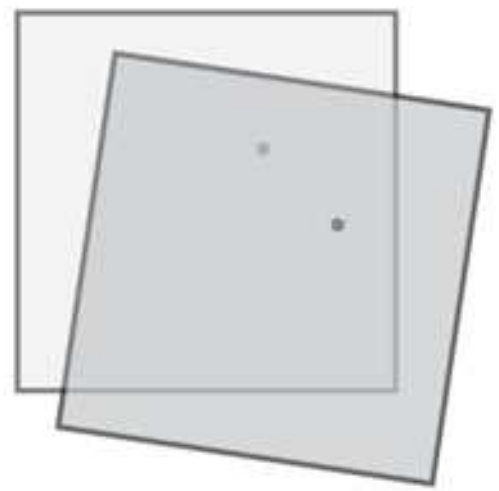

M93)

$\mathrm{T} 1=2.22 \mathrm{~s}$

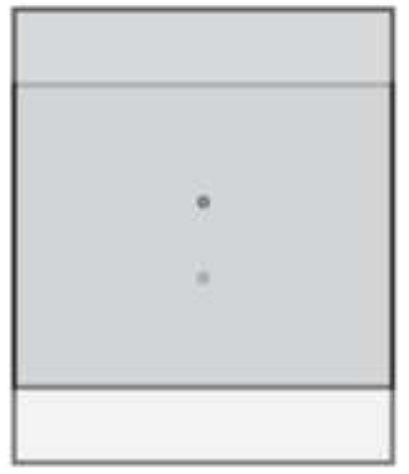

$\mathrm{T} 2-2.08 \mathrm{~s}$

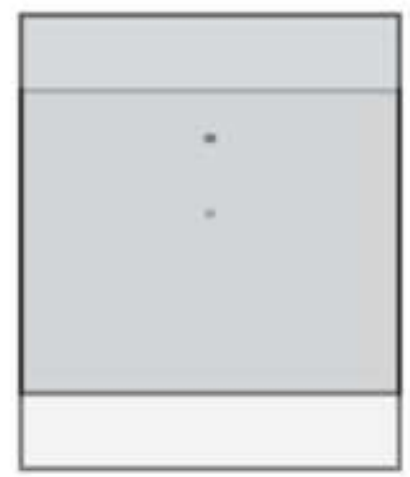

$\mathrm{T} 2=2.08 \mathrm{~s}$

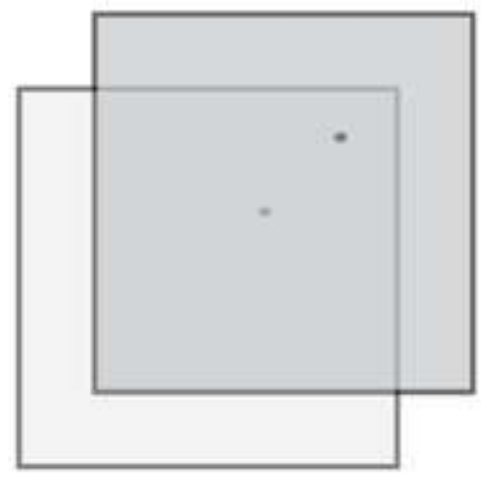

$\mathrm{T} 2=2.08 \mathrm{~s}$

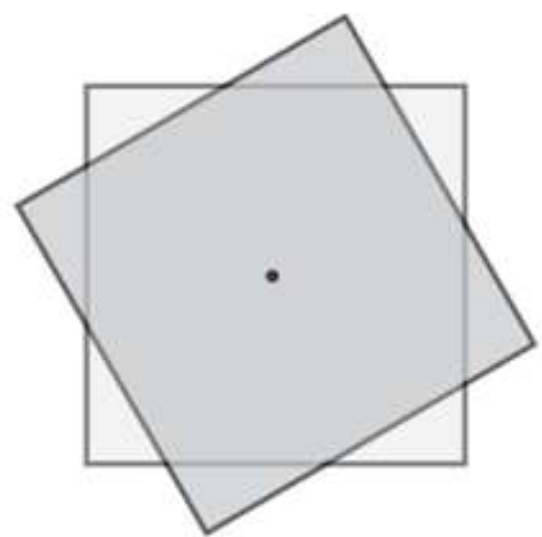

$\mathrm{T} 3=1.21 \mathrm{~s}$

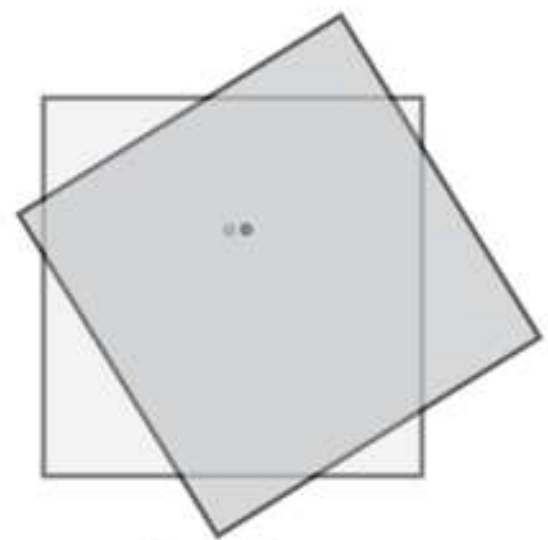

$\mathrm{T} 3=1.17 \mathrm{~s}$

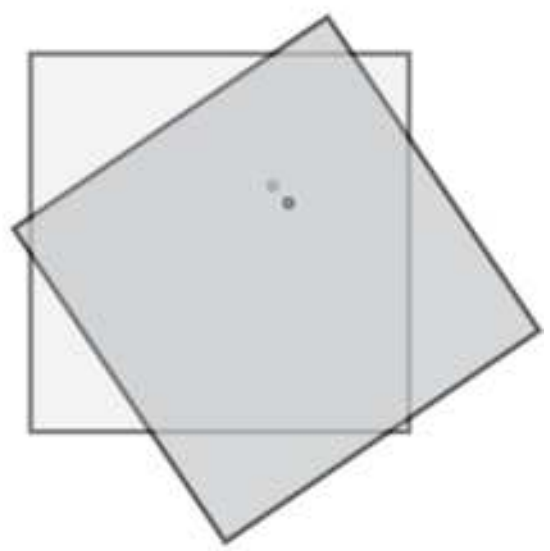

$\mathrm{T} 3=1.14 \mathrm{~s}$

Figure 2

First triple of periods and modes of vibration for M91, M92, and M93. 


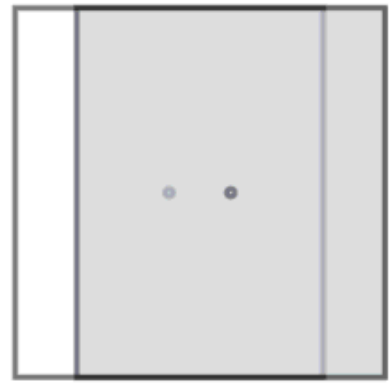

M201)

$\mathrm{T} 1=3.44 \mathrm{~s}$

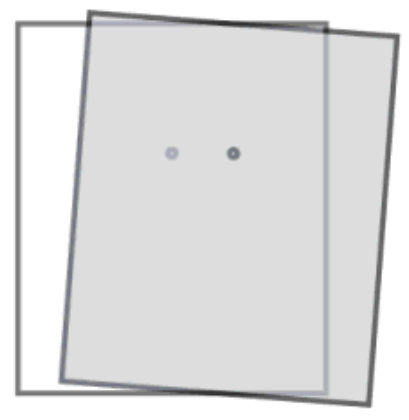

M202)

$\mathrm{T} 1=3.54 \mathrm{~s}$

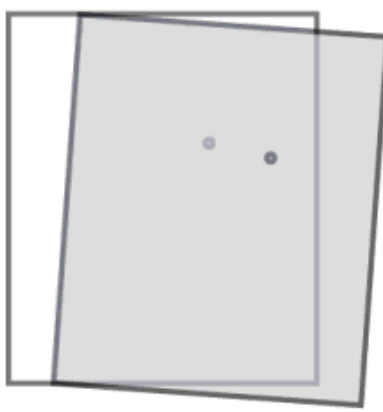

M203)

$\mathrm{T} 1=3.56 \mathrm{~s}$

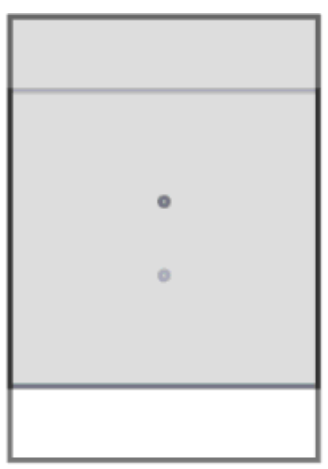

$\mathrm{T} 2=3.12 \mathrm{~s}$

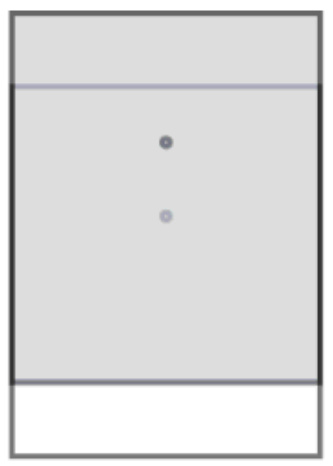

$\mathrm{T} 2=3.12 \mathrm{~s}$

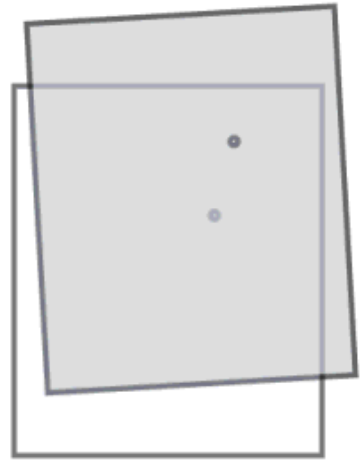

$\mathrm{T} 2=3.18 \mathrm{~s}$

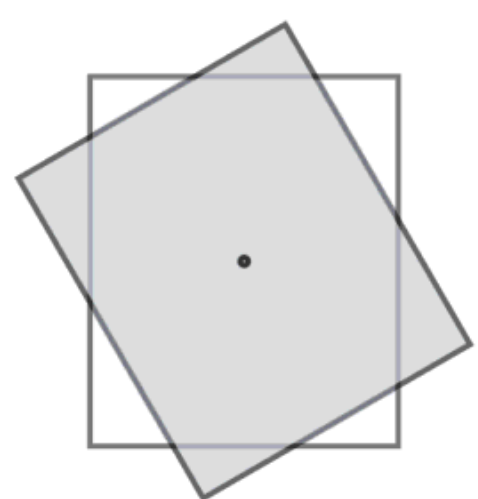

$\mathrm{T} 3=1.81 \mathrm{~s}$

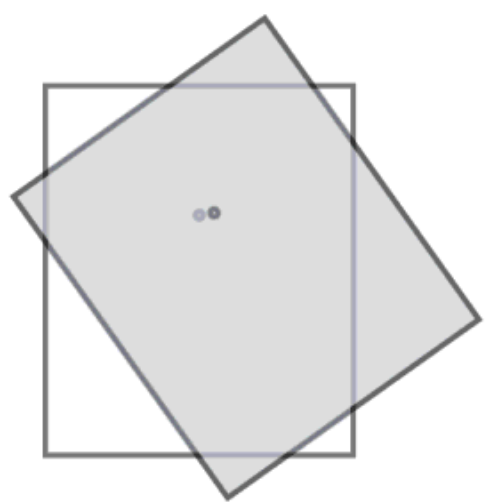

$\mathrm{T} 3=1.77 \mathrm{~s}$

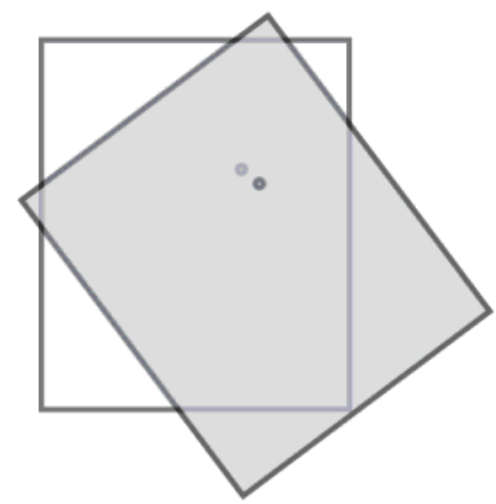

$\mathrm{T} 3=1.72 \mathrm{~s}$

Figure 3

First triple of periods and modes of vibration for M201, M202, and M203. 

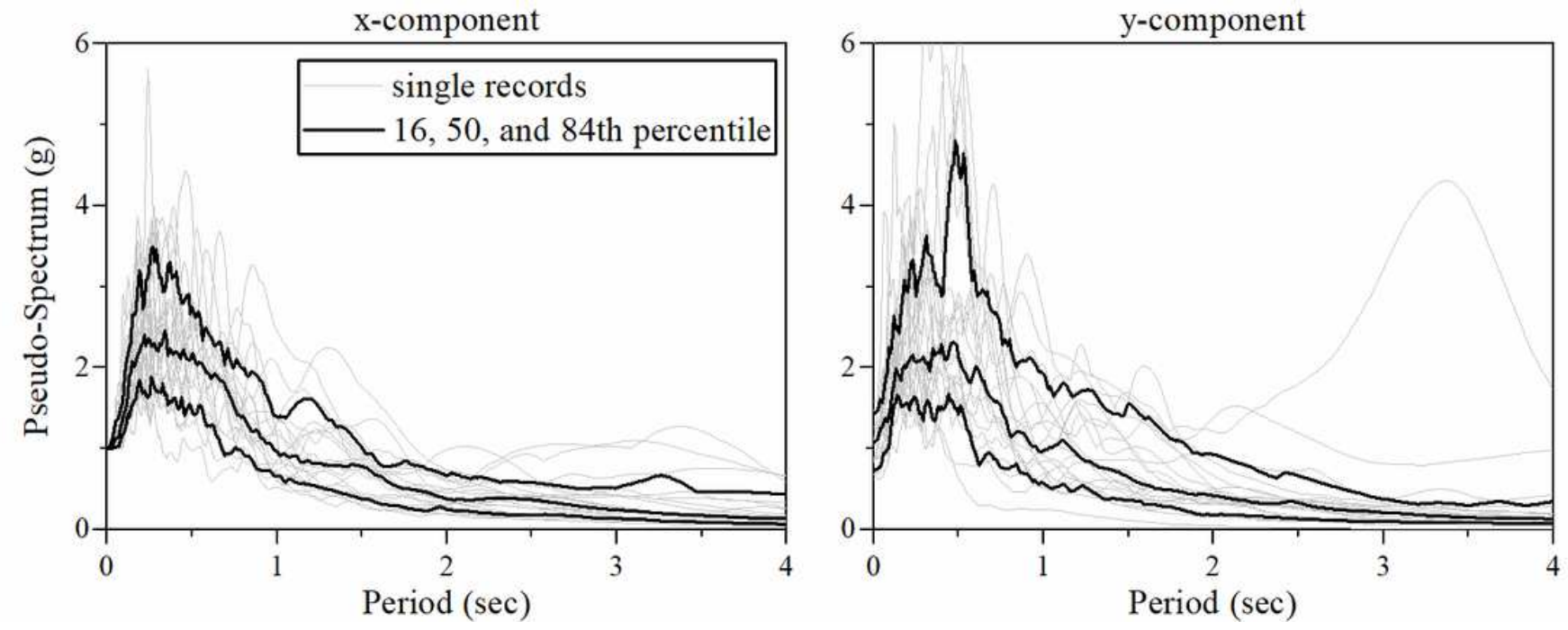

Figure 4

Pseudo-acceleration spectra for the 22 pairs of ground motion records together with their 16th, 50th, and 84th percentile spectra. 

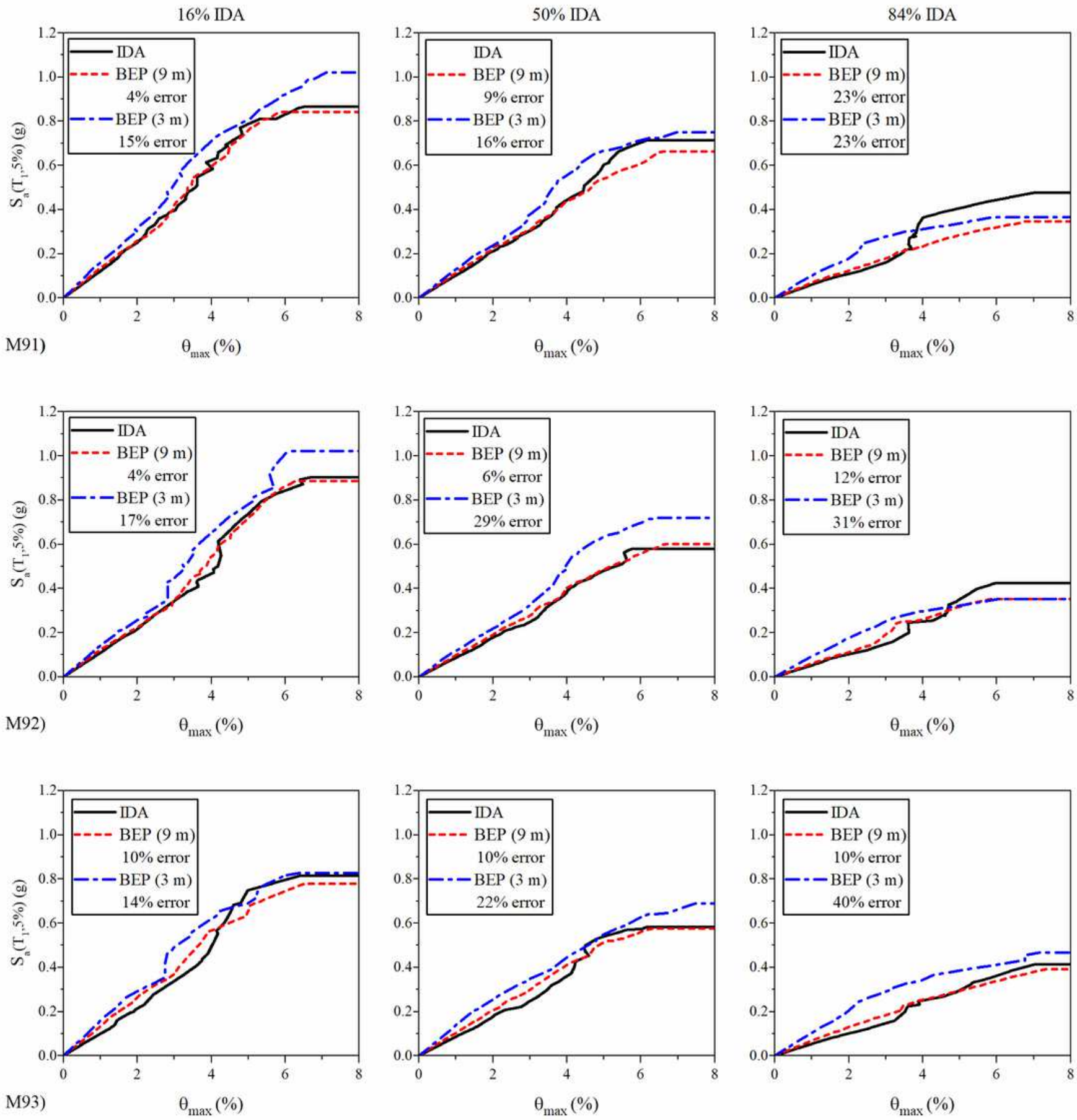

Figure 5

Sixteenth, 50th, and 84th percentile IDA curves together with the 3- and 9-mode BEP curves for M91, M92, and M93. The $x$ - and $y$-axes represent the maximum drift-ratio and IM, respectively. 

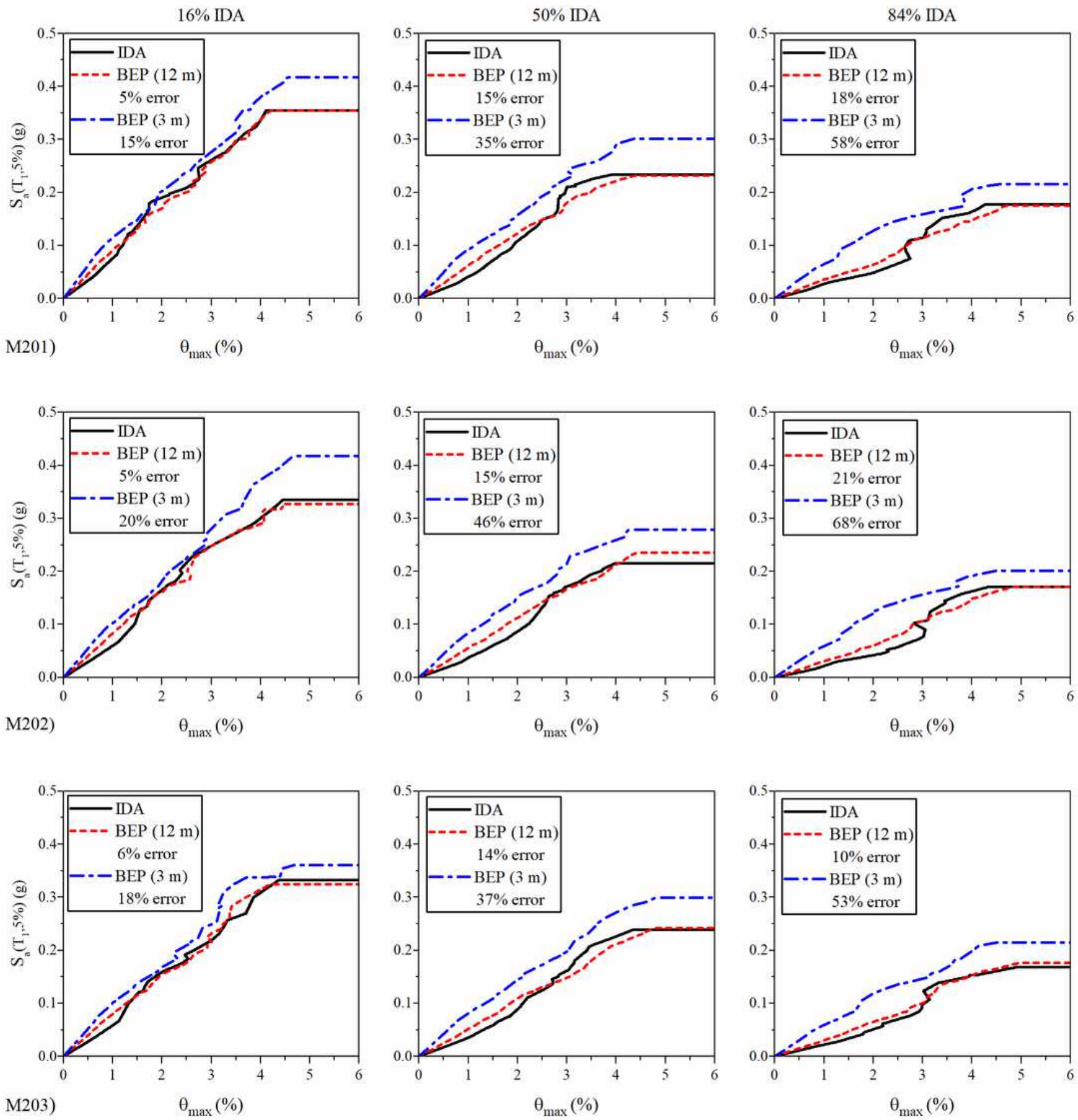

Figure 6

Sixteenth, 50th, and 84th percentile IDA curves together with the 3- and 12-mode BEP curves for M201, M202, and M203. The $x$ - and $y$-axes represent the maximum drift-ratio and IM, respectively. 

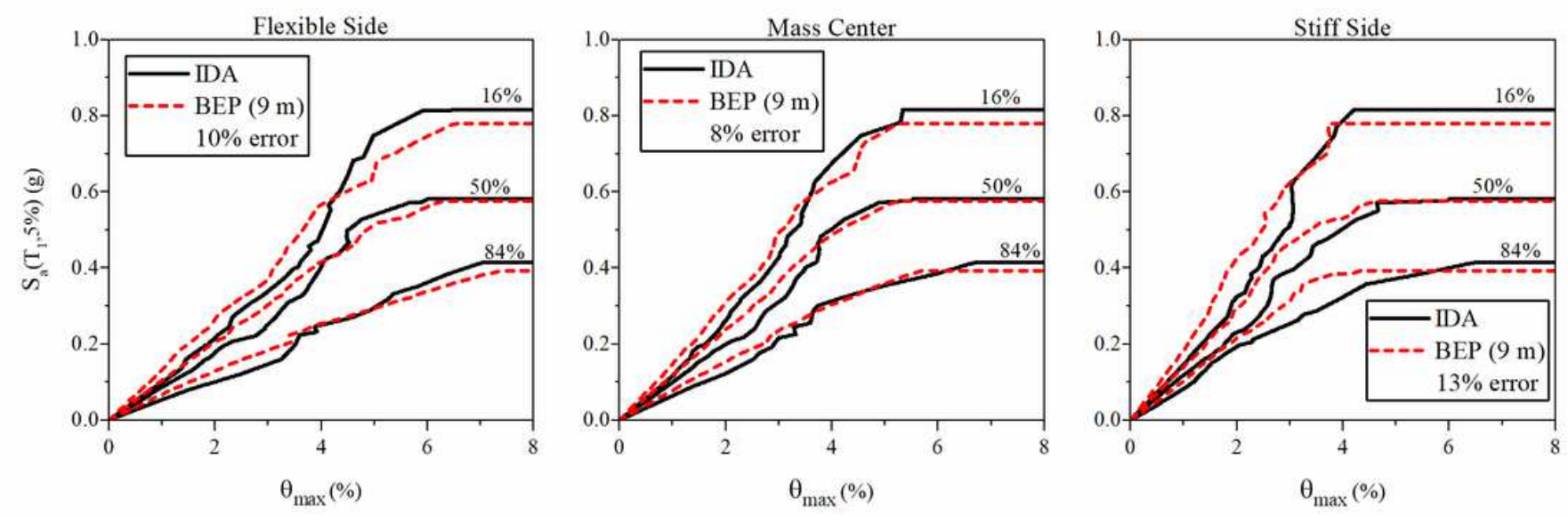

Figure 7

Sixteenth, 50th, and 84th percentile IDA curves together with the BEP curves at the 'flexible side', mass center, and 'stiff side' for M93.
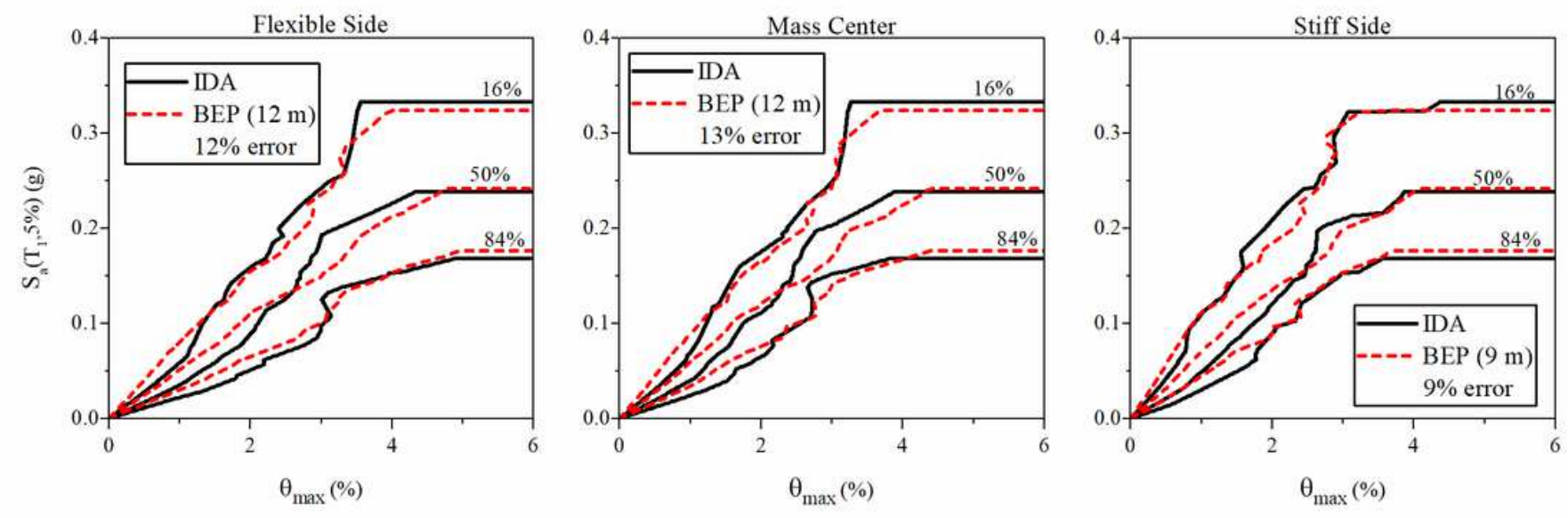

\section{Figure 8}

Sixteenth, 50th, and 84th percentile IDA curves together with the BEP curves at the 'flexible side', mass center, and 'stiff side' for M203. 

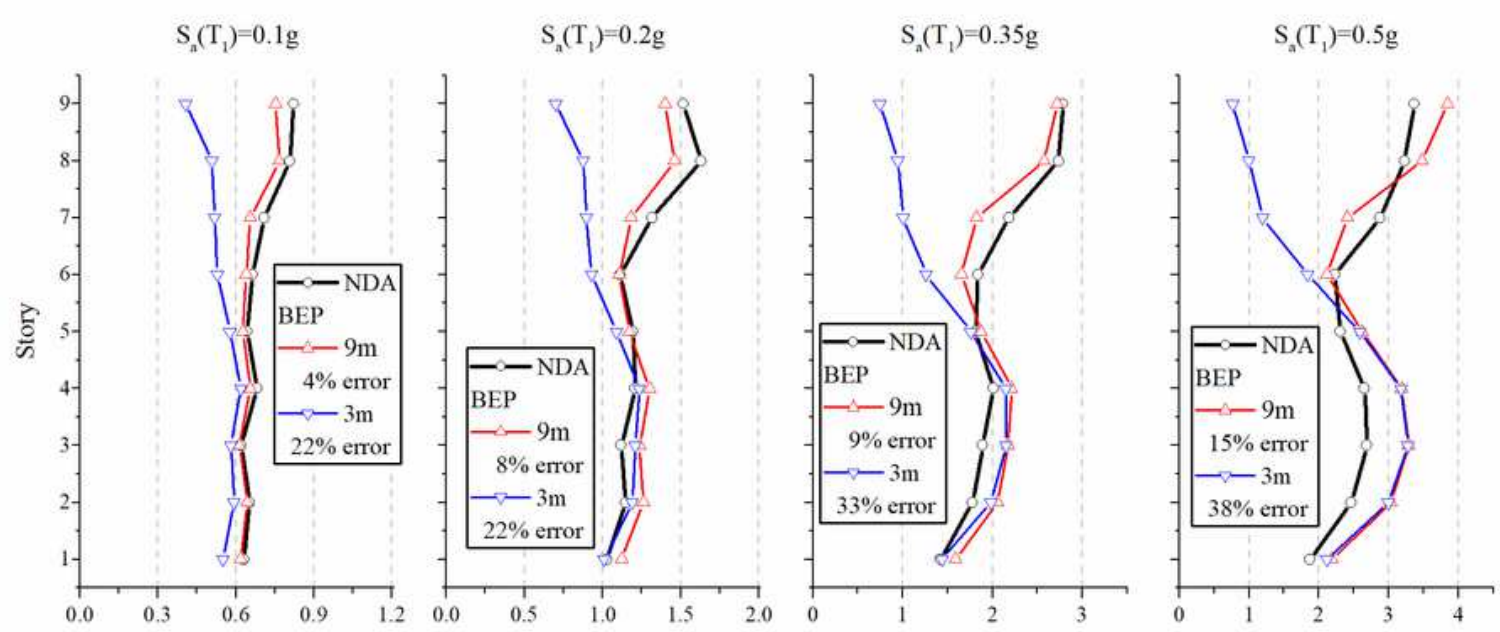

Nov
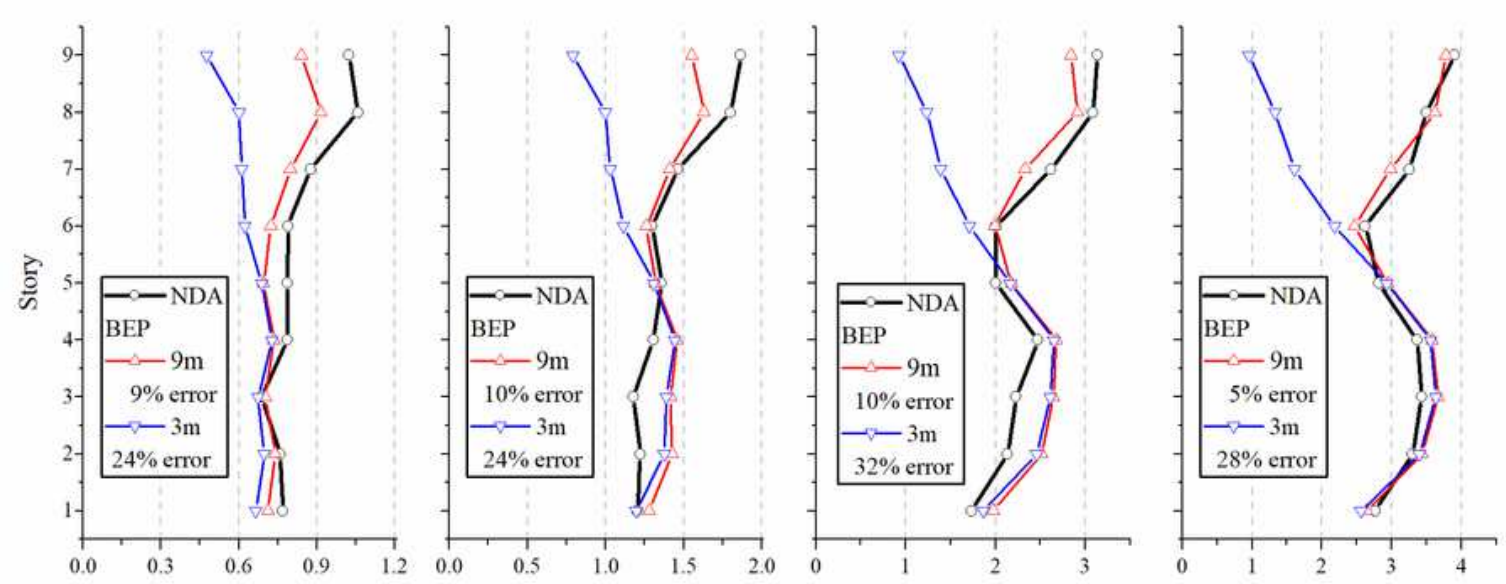

M92)
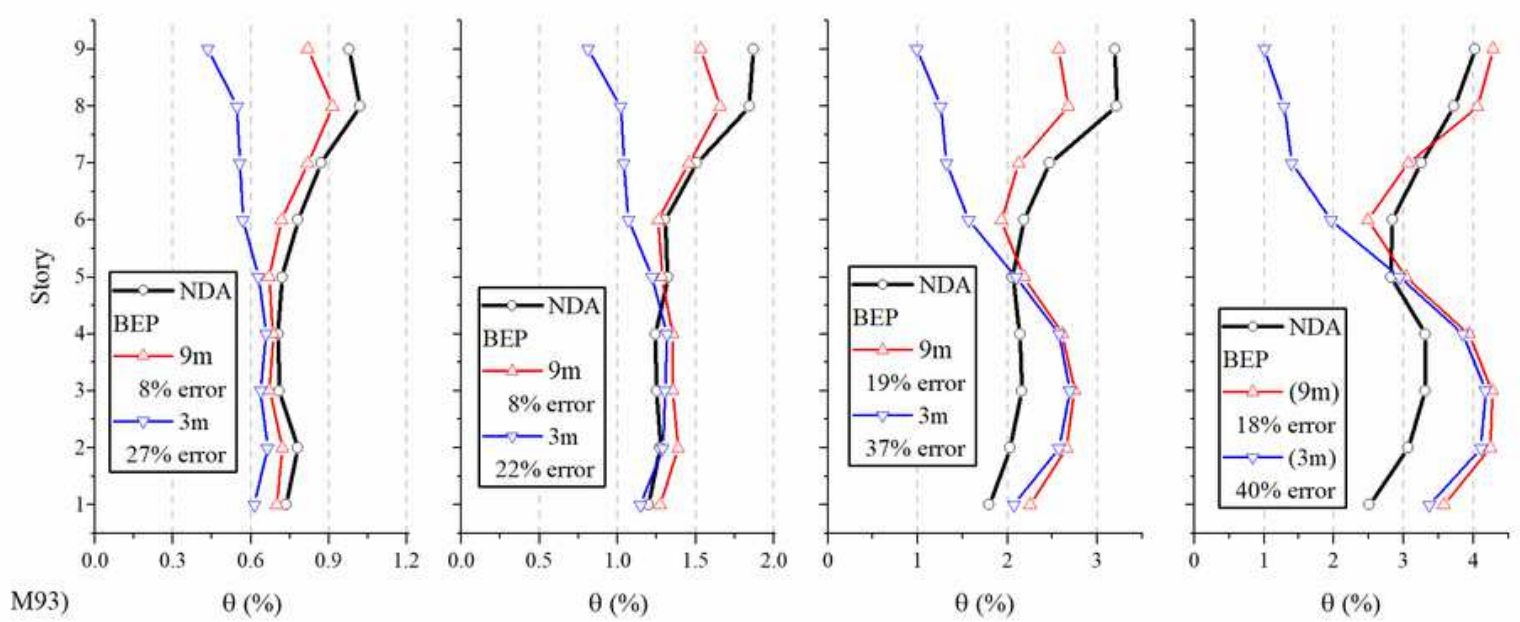

\section{Figure 9}

Median drift-ratios by NDA and BEP (3- and 9-mode) at the northern MRF of M91, M92, and M93 for the ground motion intensities $\mathrm{Sa}(\mathrm{T} 1)=0.1 \mathrm{~g}, 0.2 \mathrm{~g}, 0.35 \mathrm{~g}$, and $0.5 \mathrm{~g}$. 

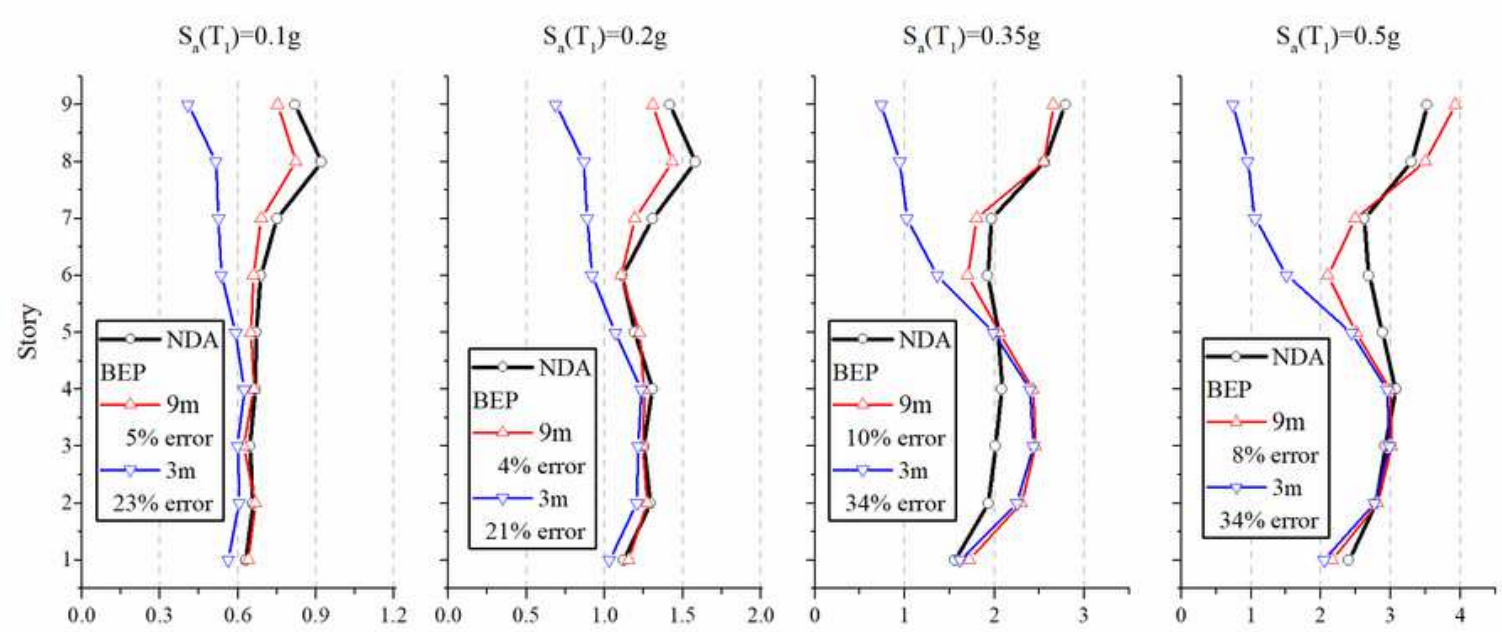

Non
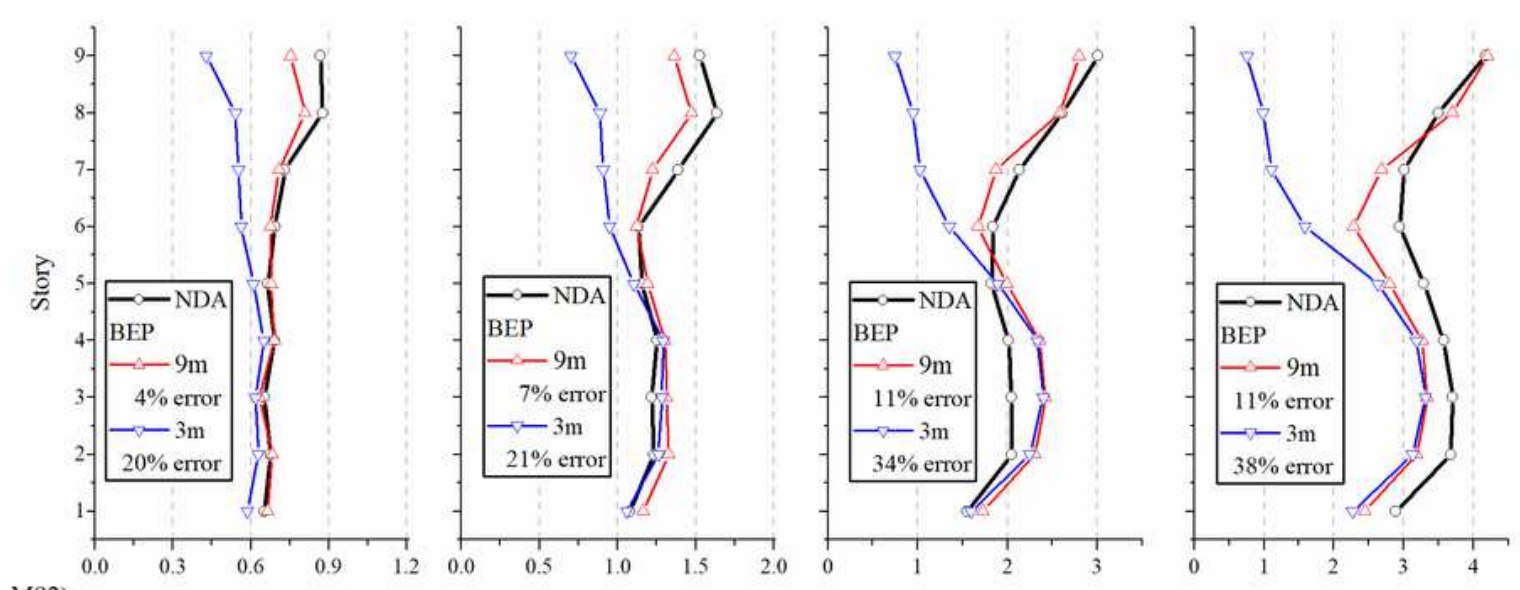

M92)
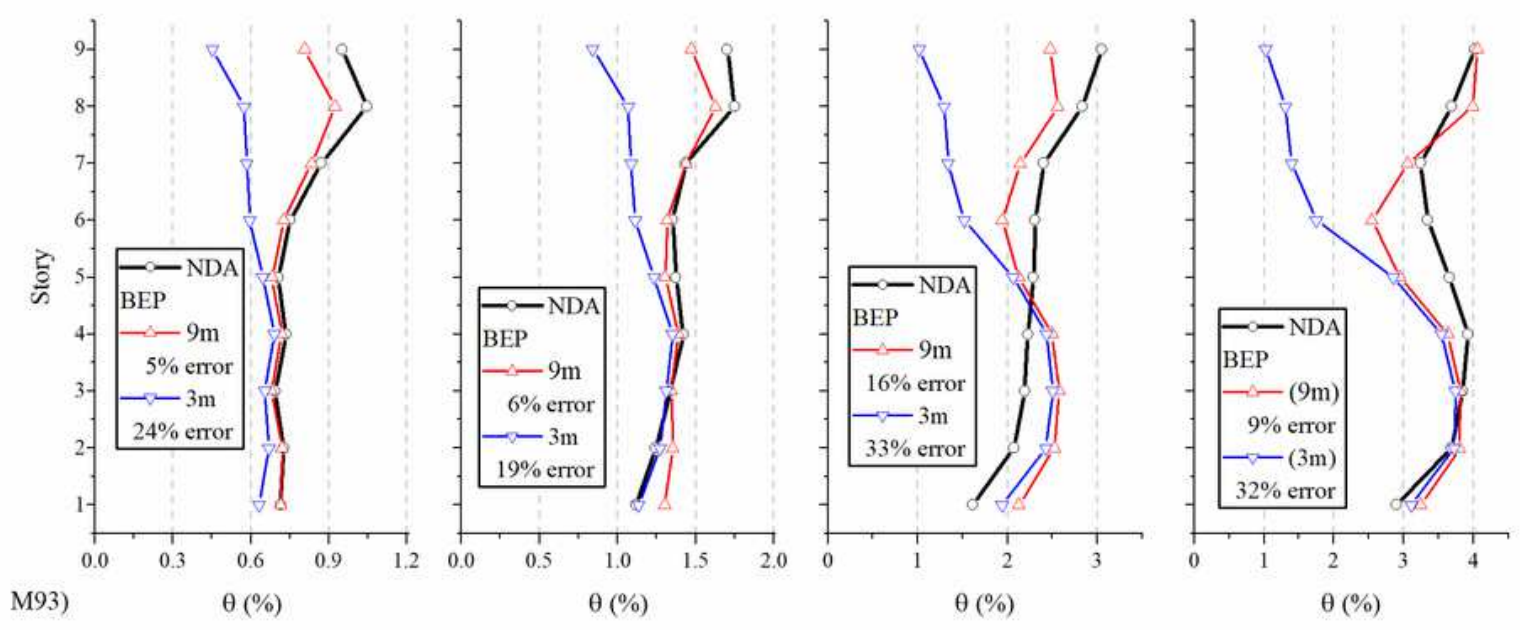

\section{Figure 10}

Median drift-ratios by NDA and BEP (3- and 9-mode) at the eastern MRF of M91, M92, and M93 for the ground motion intensities $S a(T 1)=0.1 \mathrm{~g}, 0.2 \mathrm{~g}, 0.35 \mathrm{~g}$, and $0.5 \mathrm{~g}$. 

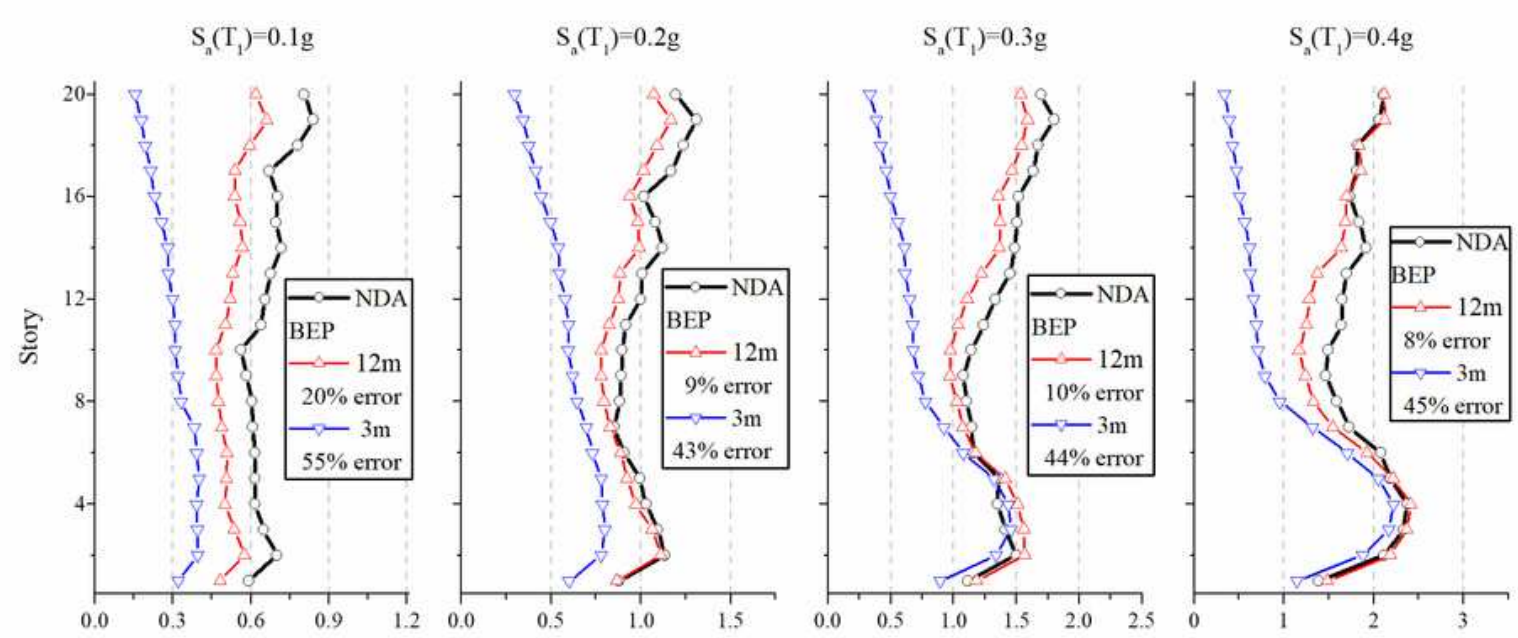

M201)
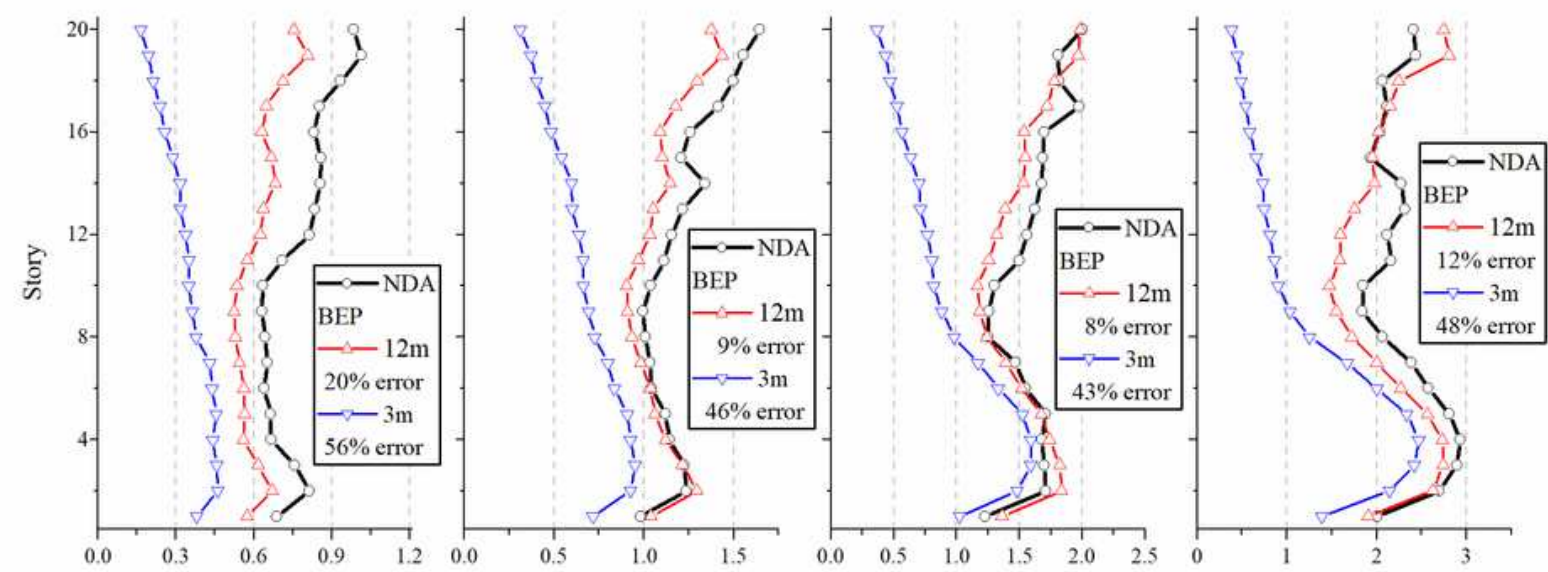

M202)
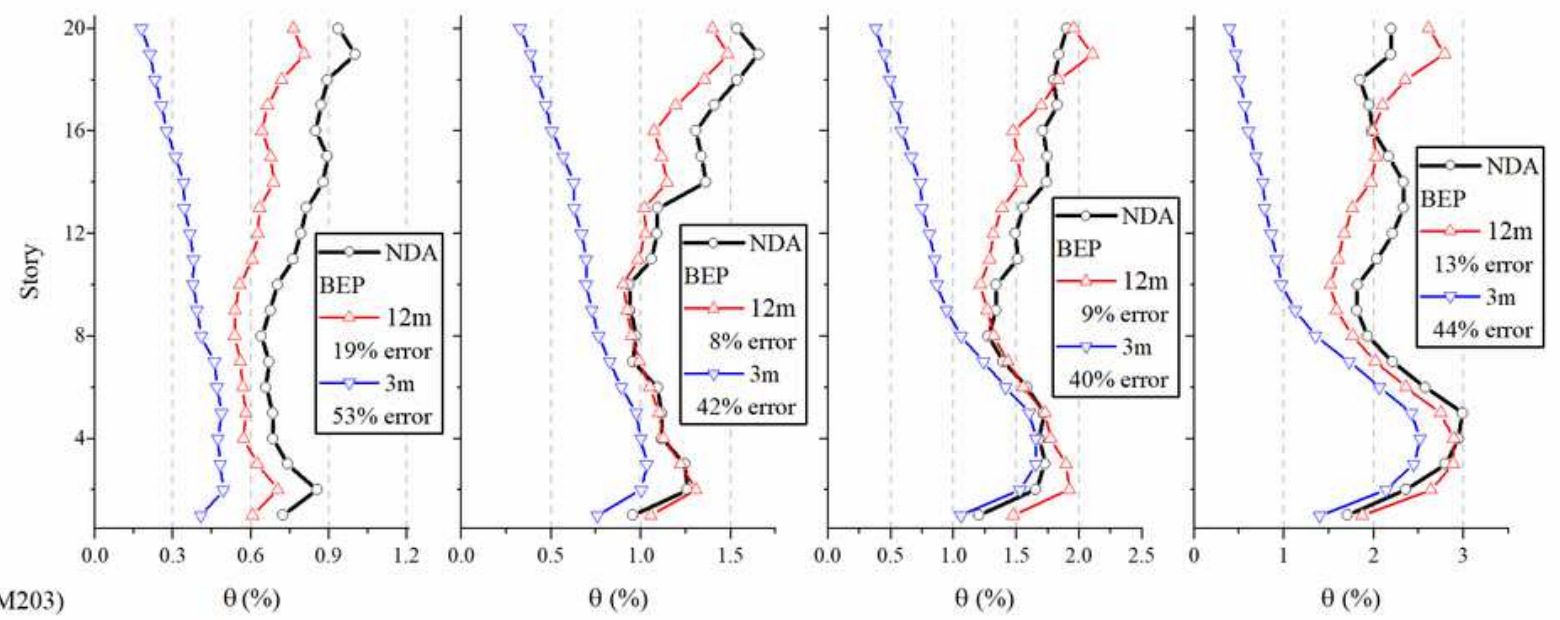

\section{Figure 11}

Median drift-ratios by NDA and BEP (3- and 12-mode) at the northern MRF of M201, M202, and M203 for the ground motion intensities $S a(T 1)=0.1 \mathrm{~g}, 0.2 \mathrm{~g}, 0.3 \mathrm{~g}$, and $0.4 \mathrm{~g}$. 

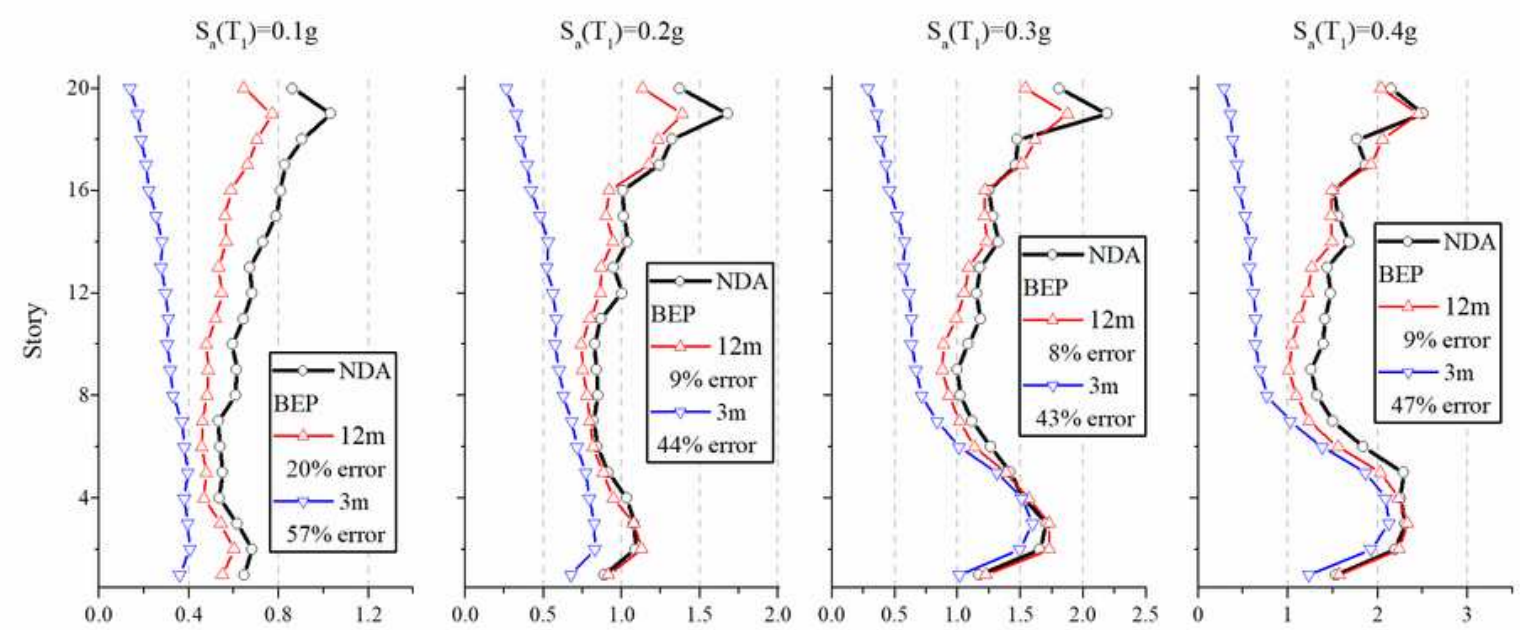

wason"
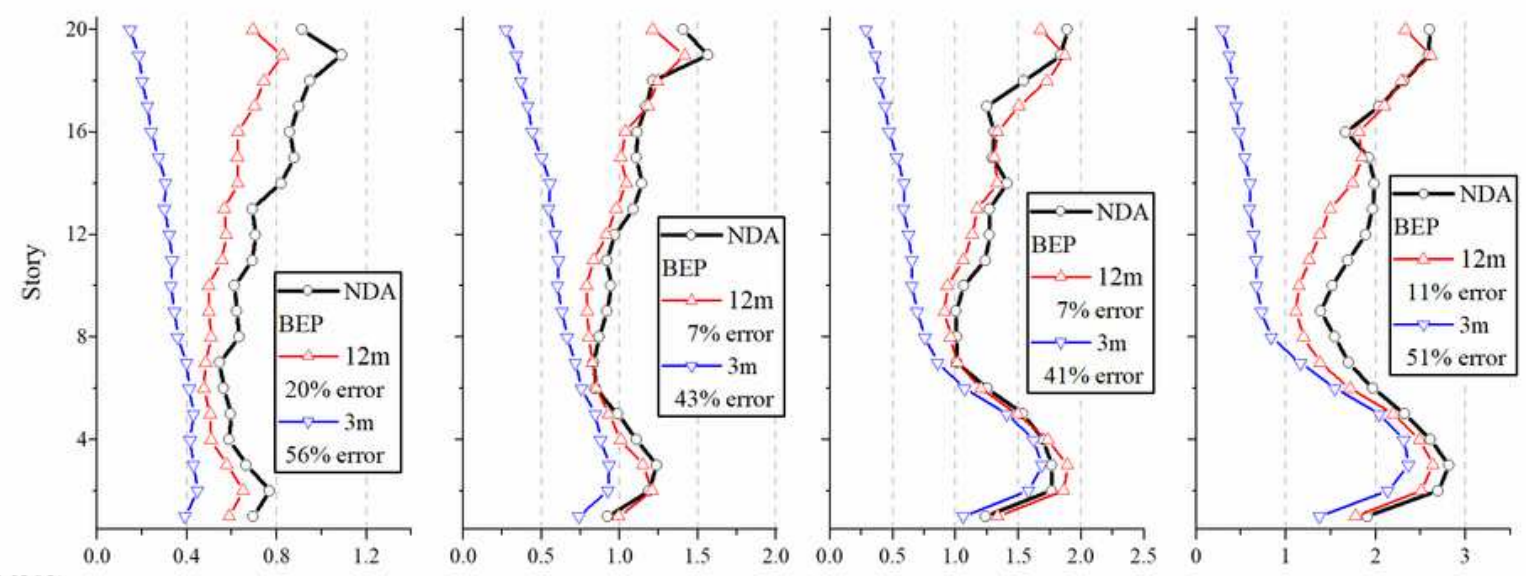

sans
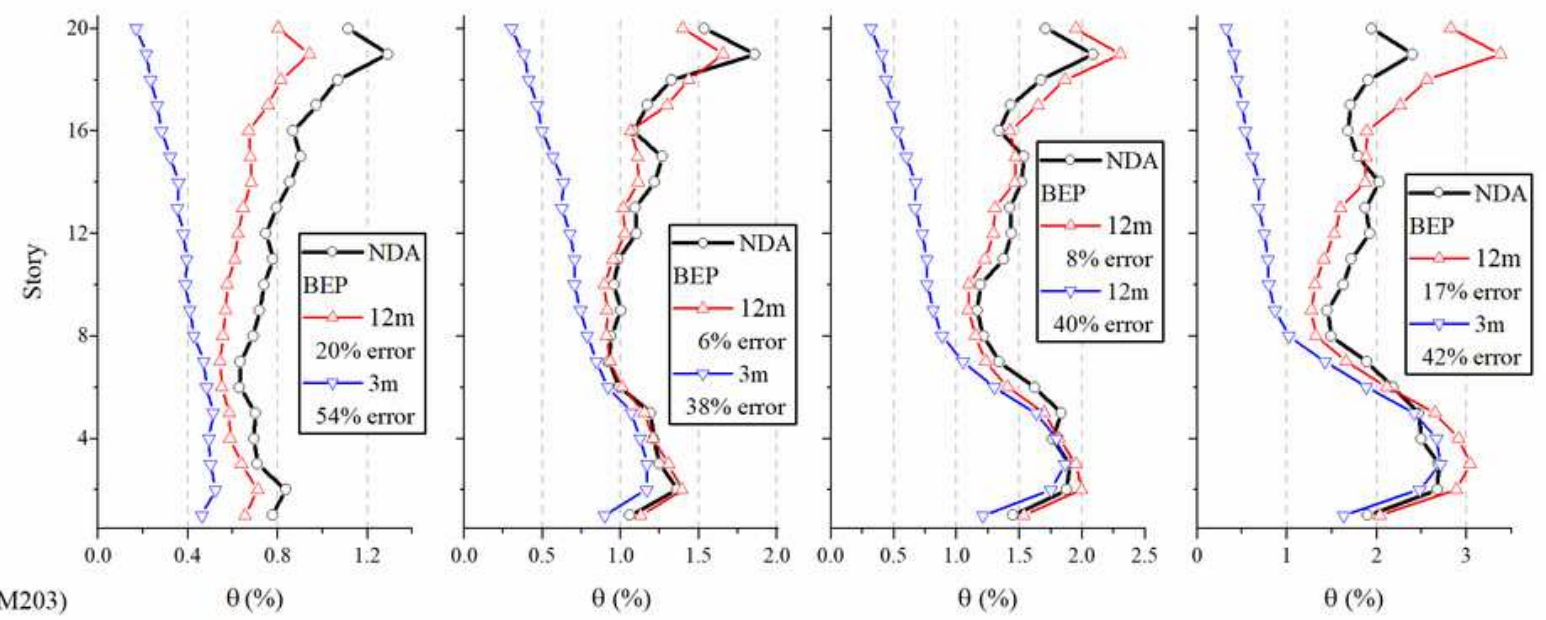

Figure 12

Median drift-ratios by NDA and BEP (3- and 12-mode) at the eastern MRF of M201, M202, and M203 for the ground motion intensities $\mathrm{Sa}(\mathrm{T} 1)=0.1 \mathrm{~g}, 0.2 \mathrm{~g}, 0.3 \mathrm{~g}$, and $0.4 \mathrm{~g}$. 